\title{
Faults in Linux 2.6
}

NICOLAS PALIX, Grenoble - Alps University/UJF, LIG-Erods GAËL THOMAS, SUMAN SAHA, CHRISTOPHE CALVÈS, GILLES MULLER and JULIA

LAWALL, Inria/UPMC/Sorbonne University/LIP6-Regal

In August 2011, Linux entered its third decade. Ten years before, Chou et al. published a study of faults found by applying a static analyzer to Linux versions 1.0 through 2.4.1. A major result of their work was that the drivers directory contained up to 7 times more of certain kinds of faults than other directories. This result inspired numerous efforts on improving the reliability of driver code. Today, Linux is used in a wider range of environments, provides a wider range of services, and has adopted a new development and release model. What has been the impact of these changes on code quality?

To answer this question, we have transported Chou et al.'s experiments to all versions of Linux 2.6, released between 2003 and 2011. We find that Linux has more than doubled in size during this period, but the number of faults per line of code has been decreasing. And the fault rate of drivers is now below that of other directories, such as arch. These results can guide further development and research efforts for the decade to come. To allow updating these results as Linux evolves, we define our experimental protocol and make our checkers available.

Categories and Subject Descriptors: D.4 [Operating Systems]: Reliability

General Terms: Reliability, Experimentation, Measurement

Additional Key Words and Phrases: Linux, fault-finding

ACM Reference Format:

Nicolas Palix, Gaël Thomas, Suman Saha, Christophe Calvès, Gilles Muller and Julia Lawall. 2013. Faults in Linux 2.6 ACM Trans. Comput. Syst. 32, 2, Article 4 (June 2014), 41 pages.

DOI : http://dx.doi.org/10.1145/2619090

\section{INTRODUCTION}

The Linux operating system is widely used, on platforms ranging from embedded systems, to personal computers, to servers and supercomputers. As an operating system (OS) with a traditional monolithic kernel, Linux is responsible for the security and integrity of the interactions between the software and the underlying hardware. Therefore, its correctness is essential. Linux also has a large developer base, as it is open source, and is rapidly evolving. Thus, it is critical to be able to continually assess and control the quality of its code.

Over 10 years ago, in 2001, Chou et al. published a study of the distribution and lifetime of certain kinds of fault 11 in OS code, focusing mostly on the x86 code in the Linux kernel [Chou et al. 2001], in versions up to 2.4.1. The ability to collect fault information automatically from such a large code base was revolutionary at the time, and this work has been highly influential. Indeed, their study has been cited over 580 times, according to Google Scholar, and has been followed by the development of a whole series of strategies for automatically finding faults in systems code [Aiken et al. 2007, Li and Zhou 2005; Searls 2004; The Kernel Janitors 2010; Wheeler 2006]. The statistics reported by Chou et al. have been used for a variety of purposes, including providing evidence that driver code is unreliable [Herder et al. 2009; Swift et al. 2006], and evidence that certain OS subsystems are more reliable than others [Depoutovitch and Stumm 2010].

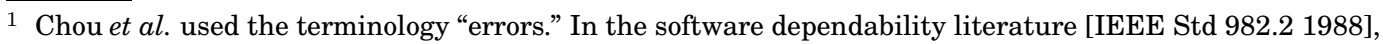
however, this term is reserved for incorrect states that occur during execution, rather than faults in the source code, as were investigated by Chou et al. and are investigated here. 
Linux, however, has changed substantially since 2001, and thus it is worth examining the continued relevance of Chou et al.'s results. In 2001, Linux was a relatively young OS, having first been released only 10 years earlier, and was primarily used by specialists. Today, well-supported Linux distributions are available, targeting servers, embedded systems, and the general public [Fedora 2013; Ubuntu 2013]. Linux code is changing rapidly, and only $30 \%$ of the code in the Linux 2.6 .33 kernel is more than five years old [Corbet 2010a; Corbet 2010b]. As of 2014, Linux supports 29 architectures, up from 13 in Linux 2.4.1 (2001) and 24 in Linux 3.0 (2011), and the developer base has grown commensurately. The development model has also changed substantially. Until Linux 2.6.0, which was released at the end of 2003, Linux releases were split into stable versions, which were installed by users, and development versions, which accommodated new features. Since Linux 2.6.0 this distinction has disappeared; releases in the 2.6 and 3 series occur every three months, and new features are made available whenever they are ready. Finally, a number of fault finding tools have been developed that target Linux code. Patches are regularly submitted for faults found using checkpatch [Checkpatch 2006], Coccinelle [Padioleau et al. 2008], Coverity [Coverity 2008], smatch [The Kernel Janitors 2010] and Sparse [Searls 2004; Sparse 2003].

In this paper, we transport the experiments of Chou et al. to the versions of Linux 2.6, in order to reevaluate their results in the context of the recent state of Linux development. Because Chou et al.'s fault finding tool and checkers were not released, and their results were released on a local web site that is no longer available, it is impossible to exactly reproduce their results on recent versions of the Linux kernel 26 To provide a baseline that can be more easily updated as new versions are released, we propose an experimental protocol based on the open source tools Coccinelle [Padioleau et al. 2008], for automatically finding faults in source code, and Herodotos [Palix et al. 2010a], for tracking these faults across multiple versions of a software project. We validate this protocol by replicating Chou et al.'s experiments as closely as possible on Linux 2.4.1 and then apply our protocol to all versions of Linux 2.6 released between December 2003 and May 2011. Finally, the next Linux version, Linux 3.0 (released July 2011), is also covered as a potential starting point for future studies. Note however that the change in version numbering is aesthetic and to celebrate the $20^{\text {th }}$ anniversary of Linux 3 , and so the difference between 2.6.39 and 3.0 is in principle no greater or lesser than the difference between any other 2.6 version and its successor. To ensure the perenity and reproducibility of our work, our tools and results are available in the appendices 4 of a research report [Palix et al. 2010c] hosted in an open archival repository.

The contributions of our work are as follows:

- We provide a repeatable methodology for finding faults in Linux code, based on open source tools, and a publicly available archive containing our complete results.

- We show that the faults kinds considered 10 years ago by Chou et al. are still relevant, because such faults are still being introduced and fixed, in both new and existing files. These fault kinds vary in their impact, but we have seen many patches for these kinds of faults submitted to the Linux kernel mailing list [LKML 2013] and have not seen any receive the response that the fault was too trivial to fix.

\footnotetext{
${ }^{2}$ Chou et al.'s work did lead to the development of the commercial tool Coverity, but using it requires signing an agreement not to publish information about its results (http://scan.coverity.com/policy.html\#license).

${ }^{3}$ See https://kml.org/lkml/2011/5/29/204 and https://kml.org/lkml/2011/7/21/455

${ }^{4}$ One may have to open the detailed view to see the appendices. The version 2 of the appendices corresponds to the data and tools of the preliminary version of this work [Palix et al. 2011]. Version 3 updates the data and tools used for this paper.
} 
- We show that while the rate of introduction of such faults continues to rise, the rate of their elimination is rising slightly faster, resulting in a kernel that is becoming more reliable with respect to these kinds of faults. This is in contrast with previous results for earlier versions of Linux, which found that the number of faults was rising with the code size.

- We show that the rate of the considered fault kinds is falling in the drivers directory, which suggests that the work of Chou et al. and others has succeeded in directing attention to driver code. The directories arch (HAL) and fs (file systems) now show a higher fault rate, and thus it may be worthwhile to direct research efforts to the problems of such code.

- We show that the lifespan of faults in Linux 2.6 is comparable to that observed by Chou et al. for earlier versions. Chou et al. observed a lifespan of 2.5 or 1.8 years according to how faults not yet fixed at the end of the considered period are taken into account. For Linux 2.6, we observe an average fault lifespan between 2.5 and 3.0 years. The Linux 2.6 median lifespan ranges between 1.5 and 2.2 years and thus has increased since the period considered by Chou et al., where the reported median was 1.25 years. However, the median age of faults has decreased by 6 months over the Linux 2.6 versions. Moreover, we find that fault kinds that are more likely to have a visible impact during execution have a much shorter average lifespan, of as little as one year.

- Although fault-finding tools are now being used regularly in Linux development, they seem to have only had a small impact on the kinds of faults we consider. Research is thus needed on how such tools can be better integrated into the development process. Our experimental protocol exploits previously collected information about false positives, reducing one of the burdens of tool use, but we propose that approaches are also needed to automate the fixing of faults, and not just the fault finding process.

The rest of this paper is organized as follows. Section 2 briefly presents our experimental protocol based on Coccinelle and Herodotos. Section 3 gives some background on the evolution of Linux. Section 4 establishes a baseline for our results, by comparing our results for Linux 2.4.1 with those of Chou et al. Section 5 presents a study of Linux 2.6, considering the kinds of code that contain faults, the distribution of faults across Linux code, and the lifetime of faults. Section [6 correlates the faults with other factors related to the Linux development model such as the activity of the developers, the structure of the code and its evolution. Section 7 evaluates the interest and applicability of the study to new code. Section 8 presents some limitations of our approach. Finally, Section 9 describes related work and Section 10 presents our conclusions. Throughout the paper, our main findings are shown in italics.

\section{EXPERIMENTAL PROTOCOL}

In the laboratory sciences there is a notion of experimental protocol, giving all of the information required to reproduce an experiment. We believe that there is a need for such a protocol in the study of software as well. For a study of faults in operating systems code, such a protocol should include the definition of the fault finding tools and checkers, as each of these elements substantially affects the results. In this section, we first present our checkers (Section 2.1), then describe how we calcuate the fault rate (Section 2.2), and finally describe the tools (Section 2.3) that we have used in the fault finding and validation process. All of our results, as well as the scripts used and the source code of the tool used, Coccinelle and Herodotos, are available on the open access archive HAL [Palix et al. 2010c] and on the publicly available project website [Palix et al. 2010b]. 


\subsection{Fault finding checkers}

The exact definitions of the checkers used by Chou et al. are not publicly available. Based on the descriptions of Chou et al., we have implemented our interpretations of their Block, Null, Var, Inull, Range, Lock, Intr, LockIntr, Float, and Size checkers. We omit the Real checker, related to the misuse of realloc, and the Param checker, related to dereferences of user-level pointers, as in both cases, we did not have enough information to define checkers that found any faults. For instance, to perform memory reallocation, there is no krealloc function in Linux 2.4.1, as there is in recent kernel versions; We tried looking for other memory reallocation function, e.g. skb_realloc_headroom, but the checker did not find any fault. In the description of each checker, the initial citation in italics is the description provided by Chou et al.

Block. "To avoid deadlock, do not call blocking functions with interrupts disabled or a spinlock held." Implementing this checker requires knowing the set of functions that may block, the set of functions that disable interrupts, and the set of functions that take spinlocks. These functions vary across Linux versions. Identifying them precisely requires a full interprocedural analysis of the Linux kernel source code, including a precise alias analysis, as these operations may be performed via function pointers. To our knowledge, Chou et al.'s tool xgcc did not provide these features in 2001, and thus we assume that these functions were identified based on their examination of the source code and possibly heuristics for collecting functions with particular properties. We take the same approach, but add a simple interprocedural analysis, based on the iterative computation of a transitive closure through the call graph. This iterative analysis implies that our Block checker automatically takes into account the new blocking functions that are added in each version.

To identify blocking functions, we consider two kinds of functions as the starting point of our interprocedural analysis. First, we observe that basic memory allocation functions, such as the kernel function kmalloc, often take as argument the constant GFP_KERNEL when they are allowed to block until a page becomes available. Thus, we consider that a function that contains a call with GFP_KERNEL as an argument may block. Second, we observe that blocking is directly caused by calling the function schedule. Given this initial list of blocking functions, we then iteratively augment the list with the names of functions that call functions already in the list without first explicitly releasing locks or turning on interrupts, until reaching a fixed point.

To identify functions that turn off interrupts and take locks, we rely on our knowledge of a set of commonly used functions for these purposes, listed in the appendix. BlockIntr checks for the case where a blocking function is called while interrupts are disabled. However, we observe that blocking with interrupts turned off is not necessarily a fault, and indeed core Linux scheduling functions, such as interruptible_sleep_on, call schedule with interrupts turned off. We have taken this issue into account when checking for false positives. Analogously, we consider the case where a blocking function is called while holding a spinlock, which is always a fault. We refer to this checker as BlockLock to highlight the different design.

Null. "Check potentially NULL pointers returned from routines." To collect a list of the functions that may return NULL, we follow the same iterative strategy as for the Block checker, with the starting point of the iteration being the set of functions that explicitly return NULL. Once the transitive closure is computed, we check the call sites of each collected function to determine whether the returned value is compared to NULL before it is used.

Var. "Do not allocate large stack variables $(>1 \mathrm{~K})$ on the fixed-size kernel stack." Our checker looks for local variables that are declared as large arrays, e.g., 1,024 or more 
elements for a char array. Many array declarations express the size of the array using macros, or even local variables, rather than explicit constants. Because Coccinelle does not expand macros, and indeed some macros may have multiple definitions across different architectures, we consider only array declarations where the size is expressed as an explicit constant.

Inull. "Do not make inconsistent assumptions about whether a pointer is NULL." We distinguish two cases: IsNull, where a null test on a pointer is followed by a dereference of the pointer, and NullRef, where a dereference of a pointer is followed by a null test on the pointer. The former is always an error, while the latter may be an error or may simply indicate overly cautious code, if the pointer can never be NULL. Still, at least one NullRef fault has been shown to allow an attacker to obtain root access [Spencer 2009]. This kind of code is unstable [Wang et al. 2013], implying that compiler optimisations can introduce vulnerabilities.

Range. "Always check bounds of array indices and loop bounds derived from user data." We recognize the functions memcpy_fromfs, copy_from_user and get_user as giving access to user data. Possible faults are cases where a value obtained using one of these functions is used as an array index, with no prior test on its value, and where some value is checked to be less than a value obtained using one of these functions, as would occur in validating a loop index.

Lock and Intr. "Release acquired locks; do not double-acquire locks (Lock)." "Restore disabled interrupts (Intr)." In early versions of Linux, locks and interrupts were managed separately: typically interrupts were disabled and reenabled using cli and sti, respectively, while locks were managed using operations on spinlocks or semaphores. In Linux 2.1.30, however, functions such as spin_lock_irq were introduced to combine locking and interrupt management. Thus, our Lock checker is limited to operators that only affect locks (spinlocks and, from Linux 2.6.16, mutexes), our Intr checker is limited to operators that only disable interrupts, and for the combined operations, we introduce a third checker, LockIntr. The considered functions are listed in the appendix.

Free. "Do not use freed memory." Like the Null checker, this checker first iteratively collects functions that always apply either kfree or some collected function to some parameter, and then checks each call to either kfree or a collected function for a use of the freed argument after the call.

Float. "Do not use floating point in the kernel." Most uses of floating point in kernel code are in computations that are performed by the compiler and then converted to an integer, or are in code that is part of the kernel source tree, but is not actually compiled into the kernel. Our checker only reports a floating point constant that is not a subterm of an arithmetic operation involving another constant, and thus may end up in the compiled kernel code.

Size. "Allocate enough memory to hold the type for which you are allocating." Because our checker works at the source code level without first invoking the $\mathrm{C}$ preprocessor, it is not aware of the sizes of the various data types on a particular architecture. We thus focus on the information apparent in the source code, considering the following two cases. In the first case, one of the basic memory allocation functions, kmalloc or kzalloc, is given a size argument involving a sizeof expression defined in terms of a type that is different from the type of the variable storing the result of the allocation. To reduce the number of false positives, the checker ignores cases where one of the types involved represents only one byte, such as char, as these are often used for allocations of unstructured data. We consider as a fault any case where there is no clear 
Table I. Assessment of the difficulty of finding and fixing faults, and the potential of a fault to cause a crash or hang at runtime

\begin{tabular}{|l|l|l|l|}
\cline { 2 - 4 } \multicolumn{1}{c|}{} & Find & Fix & Impact \\
\hline Block & Hard & Hard & Low \\
Null & Hard & Hard & Low \\
Var & Easy & Easy & Low \\
IsNull & Easy & Easy & Low \\
NullRef & Easy & Hard & Low \\
Range & Easy & Easy & Low \\
\hline
\end{tabular}

\begin{tabular}{|l|l|l|l|}
\cline { 2 - 4 } \multicolumn{1}{c|}{} & Find & Fix & Impact \\
\hline Lock & Easy & Easy & High \\
Intr & Easy & Easy & High \\
LockIntr & Easy & Easy & High \\
Free & Hard & Easy & High \\
Float & Easy & Hard & High \\
Size & Easy & Easy & High \\
\hline
\end{tabular}

relationship between the types, whether the allocated region is too large or too small. In the second case, there is an assignment where the right hand side involves taking the size of the left hand side expression itself, rather than the result of dereferencing that expression. In this case, the allocated region has the size of a pointer, which is typically significantly smaller than the size intended.

These faults vary in how easy they are to find in the source code, how easy they are to fix once found, and the likelihood of a runtime impact. Table I] summarizes these properties for the various fault kinds, based on our observations in studying the code. Faults involving code within a single function are often easy for both maintainers and tools to detect, and thus we designate these as "Easy." Finding "Hard" faults requires an interprocedural analysis to identify functions that have specific properties. Interprocedural analysis requires more effort or expertise from a maintainer, or more complexity in a tool. Fixing a fault may require only an easy local change, as in Size, where the fix may require only changing the argument of sizeof to the type of the allocated value. Cases that require creating new error handling code, such as Null, or choosing between several alternative fixes (e.g., moving a dereference or dropping an unnecessary null test), such as NullRef, are more difficult. Instances of fault kinds that entail more difficult fixes may benefit less from the use of tools, as the tool user may not have enough expertise to choose the correct fix. Finally, we indicate a low impact when a crash or hang is only likely in an exceptional condition (e.g., for Null or IsNull faults in error-handling code), and high when it is likely in normal execution.

\subsection{Assessing the fault rate}

The maximum number of faults that code can contain is the number of occurrences of code relevant to the fault, i.e. where a given kind of fault may appear. For example, the number of Block faults is limited by the number of calls to blocking functions. We follow Chou et al. and refer to these occurrences of relevant code as notes. Then,

$$
\text { fault rate }=\text { faults } / \text { notes }
$$

We find the notes associated with each of our checkers as follows. For Block, Null, and Free, a note is a call to one of the functions collected as part of the transitive closure in the fault-finding process. For Var, a note is a local array declaration. For Inull (IsNull and NullRef), a note is a null test of a value that is derefenced elsewhere in the same function. For Range and for Lock, Intr, or LockIntr, a note is a call to one of the user-level access functions or locking functions, respectively. For Size, a note is a use of sizeof as an argument to one of the basic memory allocation functions kmalloc or kzalloc when the argument is a type, or a use of sizeof where the argument is an expression. In the former case, as for the checker, we discard some cases that are commonly false positives such as when the argument to sizeof is a one-byte type such as char. Finally, we do not calculate the number of notes for Float, because we consider that every occurrence of a float in a context where it may be referenced in the compiled code is a fault, and thus the number of notes and faults is the same. 


\subsection{Tools}

Our experimental protocol relies on two open-source tools: Coccinelle (v1.0.0rc15), to automatically find potential faults and notes in the Linux kernels [Padioleau et al. 2008], and Herodotos (v0.6.1-rc1), to correlate the fault reports between versions [Palix et al.2010a]. We store the resulting data in a PostgreSQL database (v9.1.5), and analyze it using SQL queries.

Coccinelle performs control-flow based pattern searches in $\mathrm{C}$ code. It provides a language, the Semantic Patch Language (SmPL), for specifying searches and transformations and an engine for performing them. Coccinelle's strategy for traversing controlflow graphs is based on the temporal logic CTL [Brunel et al. 2009], while that of the tool used by Chou et al. is based on automata. There are technical differences between these strategies, but we do not expect that they are relevant here.

A notable feature of Coccinelle is that it does not expand preprocessor directives. We have only found this feature to be a limitation in the Var case, as noted in Section 2.1. On the other hand, this feature has the benefit of making the fault-finding process independent of configuration information, and thus we can find faults across the entire Linux kernel source tree, rather than being limited to a single architecture.

To be able to understand the evolution of faults in Linux code, it is not sufficient to find potential faults in the code base; we must also understand the history of individual fault occurrences. To do so, we must be able to correlate potential fault occurrences across multiple Linux versions, even in the presence of code changes in the files, and manage the identification of these occurrences as real faults and false positives. For these operations, we use Herodotos. To correlate fault occurrences, Herodotos first uses diff to find the changes in each pair of successive versions of a file for which Coccinelle has produced fault reports. If a pair of reports in these files occurs in the unchanged part of the code, at corresponding lines, the reports are automatically considered to represent the same fault, with no user intervention. Otherwise, in a pair of reports, if the report in the older version occurs in the changed part of the code, then its status is considered to be unknown, and the user must indicate, via an interface based on the emacs "org" mode [Org 2013], whether the pair to which it belongs represents the same fault or unrelated ones. We have validated this approach by manually inspecting a randomly chosen set of automatic correlations. None of the hundreds of correlations inspected was suspicous among the more than 46,000 automatic correlations performed. Once the correlation process is complete, a similar interface is provided to allow the user to classify each group of correlated reports as representing either a fault or a false positive. Further details about the process of using Herodotos are provided elsewhere [Palix et al. 2010a].

Once the fault reports are correlated and assessed for false positives, we import their histories into a database, along with the associated notes. The database also contains information about Linux releases such as the release date and code size, and information about Linux files (size, number of modifications between releases) and functions (starting and ending line numbers). The complete database, including both the reports and the extra information, contains $2 \mathrm{~GB}$ of data. To analyze the collected data, we wrote over 2,100 lines of PL/pgSQL and SQL queries and about 300 lines of $\mathrm{R}$ that extract and correlate information.

Extending the results to new versions. A benefit of our experimental protocol is that it makes it easy to extend the results to new versions of Linux. When a new version of Linux is released, it is only necessary to run the checkers on the new code, and then repeat the correlation process. As our collected data contains information not only about the faults that we have identified, but also about the false positives, Herodotos 


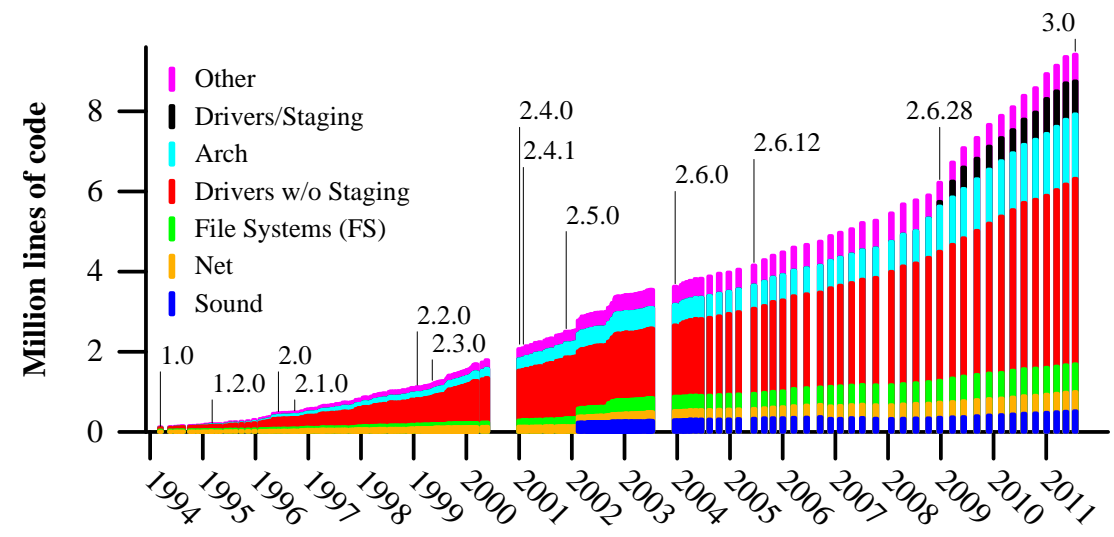

Fig. 1. Linux directory sizes (in MLOC)

automatically annotates both faults and false positives left over from previous versions as such, leaving only the new reports to be considered by the user.

\section{EVOLUTION OF LINUX}

To give an overview of the complete history of Linux, we first consider the evolution in code size of the Linux kernel between version 1.0, released in March 1994, and version 3.0, released in July 2011, as shown in Figure11, Prior to Linux 2.6, this figure shows the size of the development versions (with odd version number), when available, as new code is added in these versions, and this added code is then maintained in the subsequent stable versions (with even version number). Starting with Linux 2.6, we show all major versions, all of which can contain new code. Code sizes are computed using David A. Wheeler's SLOCCount (v2.26) [Wheeler 2013] and include only the ANSI C code. The code sizes are broken down by directory, highlighting the largest directories: drivers/staging, arch, drivers, fs (file systems), net, and sound. Drivers/staging was added in Linux 2.6.28 as an incubator for new drivers that are not yet mature enough to be used by end users. Code in drivers/staging is not compiled as part of the default Linux configuration, and is thus not included in standard Linux distributions. Sound was added in Linux 2.5.5, and contains sound drivers that were previously in the drivers directory. The largest directory is drivers, which, including drivers/staging, has made up 57\% of the source code since Linux 2.6.30.

Due to the large increase in size and the high level of churn, relatively few lines of Linux 3.0 have been present in the Linux kernel since Linux 2.6.0, as shown by Figure 2. Indeed, as reported by the linetags tool from the gitdn 5 toolsuite, only $20 \%$ of the lines of Linux 2.6.0 have been kept untouched througout the Linux 2.6 history. Except for Linux 2.6.0, every version of Linux 2.6 has contributed as new code less than $5 \%$ of the code found in Linux 3.0. Nevertheless, recent versions tend to introduce a larger part of Linux 3.0 as compared to older versions. Indeed, each version released after 2007 introduces more than $2 \%$ of the Linux 3.0 code, i.e., more than $55 \%$ in total, whereas each earlier version, except 2.6.0, introduces less than $2 \%$. Linux 2.6.0 is an exception as it represents in part the code that is inherited from the Linux 2.5 era,

\footnotetext{
${ }^{5}$ The original version of linetags is available at git:/git.lwn.net/gitdm.git and was developed by Jonathan Corbet, LWN. We made a modified version (https://github.com/npalix/gitdm) to support Linux versions prior to 2.6.12. This latter was applied on a Linux repository that aggregates linux-history and linux-2.6 thanks to a git graft point between commits 1da177e4c3f41524e886b7f1b8a0c1fc7321cac2 and e7e173af42dbf37b1d946f9ee00219cb3b2bea6a.
} 


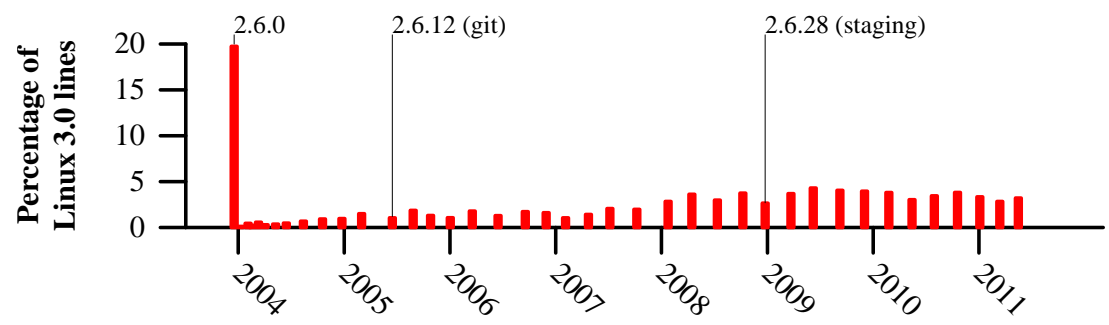

Fig. 2. Distribution of the lines of code of Linux 3.0 by the Linux 2.6 version in which they were introduced

and as we use it as the starting point for the origin of lines. Most of the code that has remained unchanged since 2.6.0 comes from driver code for old hardware (drivers), old hardware platforms (arch) and old network protocols that no longer change (net), with respectively $65.6 \%, 18.8 \%$ and $12.2 \%$ of the Linux 2.6 .0 code that is still present in Linux 3.0.

Figure 1 shows that, for most directories, the code growth has been roughly linear since Linux 1.0. Some exceptions are highlighted in Figure 3, which shows the percentage code size increase in each directory from one version to the next. We have marked some of the larger increases and decreases. Many of the increases involve the introduction of new services (e.g., ieee802.11 for wireless support in version 2.6.14), and new file systems (e.g., the Btrfs filesystem in version 2.6.29). In Linux 2.6.19 and 2.6.23, old OSS drivers already supported by ALSA were removed from sound, decreasing its size. In Linux 2.6.27, arch was reorganized, and received some large header files from include, adding around 180,000 lines of $\mathrm{C}$ code. The most recent decrease is in the $\mathrm{fs}$ directory of Linux 2.6.37, where the Ceph distributed file system was reorganized and partly moved to the net directory. Another decrease, at the same time, is due to the Server Message Block File System (SMBFS), which is on its way out of the kernel, as it has been superseded by the Common Internet File System (CIFS). Finally, staging grew substantially in 2.6.29 and 2.6.37. All in all, these changes have resulted in code growth from 2 MLOC in 2001 to more than 9 MLOC in 2011.

In our study, we are less interested in the absolute number of lines of code than the amount of code relevant to our fault kinds, i.e. notes. As shown in Figure 4 , the increase in code size has induced an similar increase in the number of notes, as defined in Section 2.2, in almost all cases. In fact, across all of Linux 2.6, the number of notes per line of code is essentially constant, between 0.027 and 0.030 and the number of lines and the number of notes are highly correlated: 0.998 by the Pearson's correlation coefficient. $[$ ]

\section{FAULTS IN LINUX 2.4.1}

Linux 2.4.1 was the latest version of Linux considered by Chou et al. [Chou et al. 2001]. To validate our experimental protocol, we have used our checkers to find faults and notes in this version, and we compare our results to those provided in their paper. We focus on the results that are specific to Linux 2.4.1, rather than those that relate to the history of Linux up to that point, to avoid the need to study earlier versions that are of little relevance today.

${ }^{6}$ This coefficient ranges from -1 to 1 inclusive, with 0 indicating that the two variables are not correlated, and an absolute value of 1 , that the variable are correlated. 


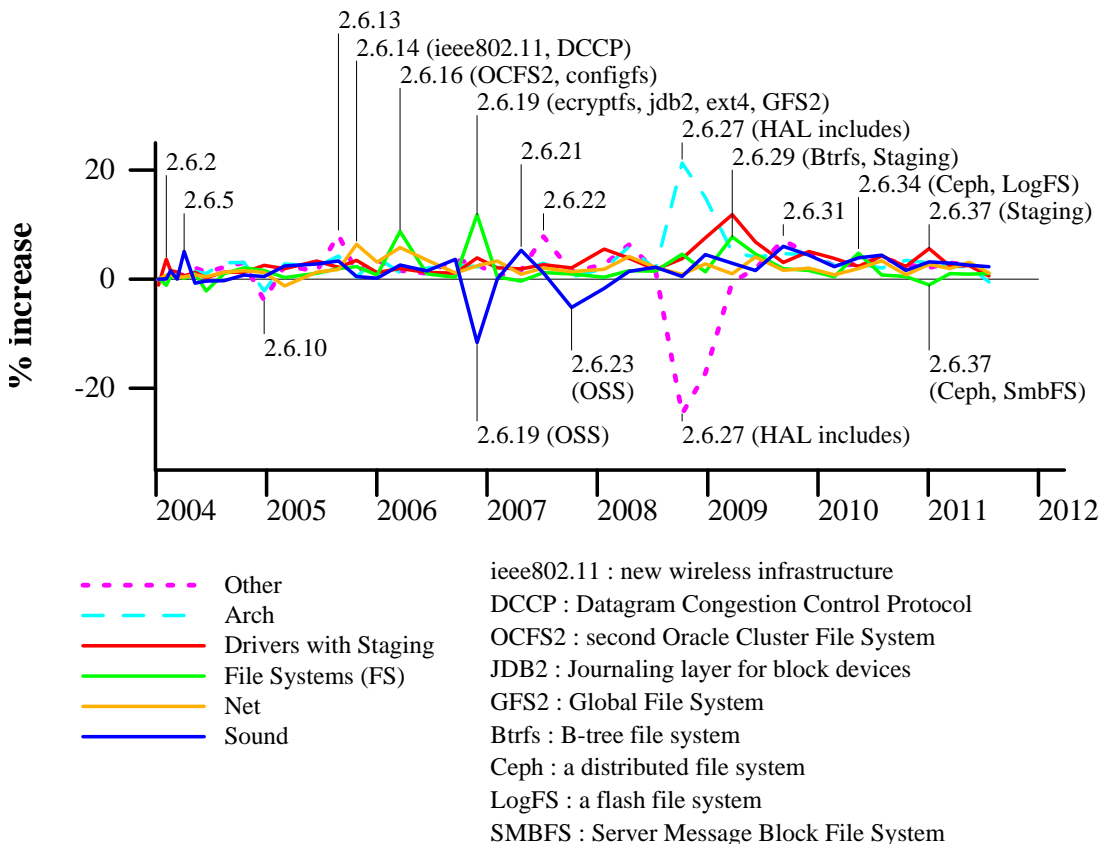

Fig. 3. Linux directory size increase

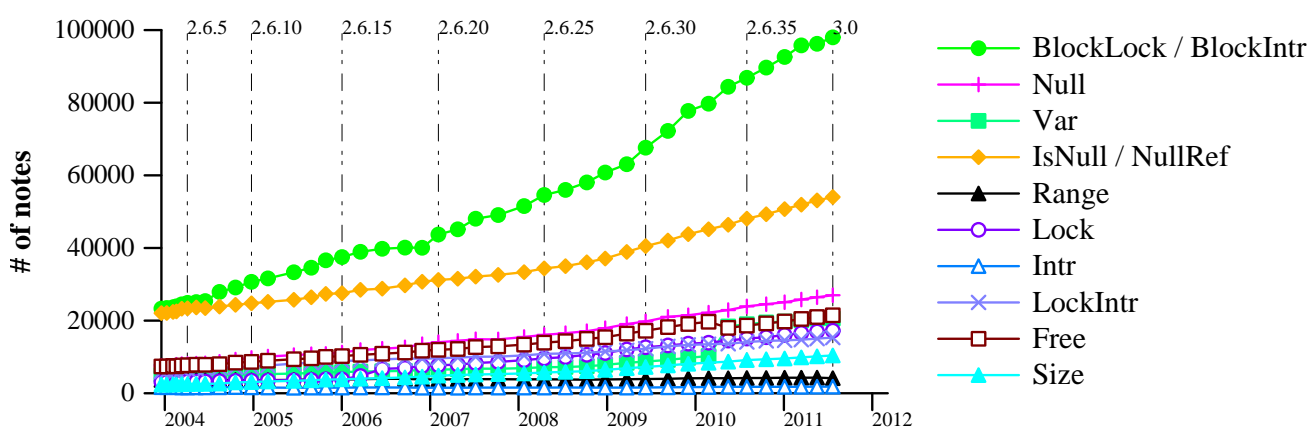

Fig. 4. Notes through time per kind

\subsection{What code is analyzed?}

For the results of fault finding tools to be comparable, the tools must be applied to the same code base. Chou et al. focus only on x86 code, finding that $70 \%$ of the Linux 2.4.1 code is devoted to drivers. Nevertheless, we do not know which drivers, file systems, etc. were included. To calibrate our results, we use SLOCCount to obtain the number of lines of ANSI $\mathrm{C}$ code in the Linux kernel and in the drivers directory, considering the three possibilities reported in Table II all code in the Linux 2.4.1 kernel source tree ("All code"), the set of . c files compiled when using the default x86 configuration ("Min $\mathrm{x} 86$ ") $]$ and all 2.4.1 code except the arch and include subdirectories that are specific to non-x86 architectures ("Max x86"). Max x86 gives a result that is closest to

\footnotetext{
${ }^{7}$ This configuration was automatically generated using make menuconfig without any modification to the proposed configuration. To collect the .c files, we compiled Linux 2.4.1 according to this configuration using a Debian 3.1 (Sarge) installation in a virtual machine, with gcc version 2.95.4 and make version 3.80.
} 
that of Chou et al., although the proportion of driver code is slightly higher than $70 \%$. This is reasonable, because some driver code is associated with specific architectures and cannot be compiled for x86. Nevertheless, these results show that we do not know the precise set of files used in Chou et al.'s tests.

\begin{tabular}{|c|c|c|c|}
\hline & All code & Min x86 & Max x86 \\
\hline Drivers LOC & $1,248,930$ & 71,938 & $1,248,930$ \\
\hline Total LOC & $2,090,638$ & 174,912 & $1,685,265$ \\
\hline Drivers \% & $59 \%$ & $41 \%$ & $74 \%$ \\
\hline
\end{tabular}

In our experiments, we consider the entire kernel source code, and not just the code for $\mathrm{x} 86$, as every line of code can be assumed to be relevant to some user.

\subsection{How many faults are there?}

For the entire Linux 2.4.1 kernel, using the checkers described in Section2.1, we obtain about 700 reports, of which we have determined that 569 represent faults and the remainder represent false positives. Chou et al.'s checkers find 1,025 faults in Linux 2.4.1. They have checked 602 of these reports; the remainder are derived from what they characterize as low false positive checkers. We have checked all of the reports included in our study.

Table III compares the number of faults found per checker. In most cases, we find fewer faults. This may be due to different definitions of the checkers, or different criteria used when identifying false positives. In the case of Var, we find fewer faults because we consider only cases where the size is explicitly expressed as a number. In a few cases, we find more faults. For example, Chou et al.'s Inull checker can be compared to our IsNull and NullRef checkers. We find fewer IsNull faults than their Inull faults, but far more NullRef faults. We also find slightly more Free faults. This may derive from considering a larger number of files, as we have found that only one of our Free faults occurs in a file that is compiled using the default x86 configuration. Results from Chou et al.'s checkers were available at a web site interface to a database, but Chou has informed us that this database is no longer available. Thus, it is not possible to determine the precise reasons for the observed differences.

Table III. Comparative fault count

\begin{tabular}{|l|l|l|l|}
\hline Checker & \multicolumn{2}{|c|}{ Chou et al. } & \multirow{2}{*}{ Our results } \\
& checked & unchecked & \\
\hline Block & 206 & 87 & N/A \\
BlockLock & N/A & N/A & 43 \\
BlockIntr & N/A & N/A & 102 \\
Null & 124 & 267 & 98 \\
Var & 33 & 69 & 13 \\
\hline Inull & 69 & 0 & N/A \\
IsNull & N/A & N/A & 36 \\
NullRef & N/A & N/A & 221 \\
\hline Range & 54 & 0 & 11 \\
Lock & 26 & 0 & 5 \\
Intr & 27 & 0 & 2 \\
LockIntr & N/A & N/A & 6 \\
Free & 17 & 0 & 21 \\
\hline Float & 10 & 15 & 8 \\
Size & 3 & 0 & 3 \\
\hline \hline Total & 569 & 438 & 569 \\
\hline
\end{tabular}




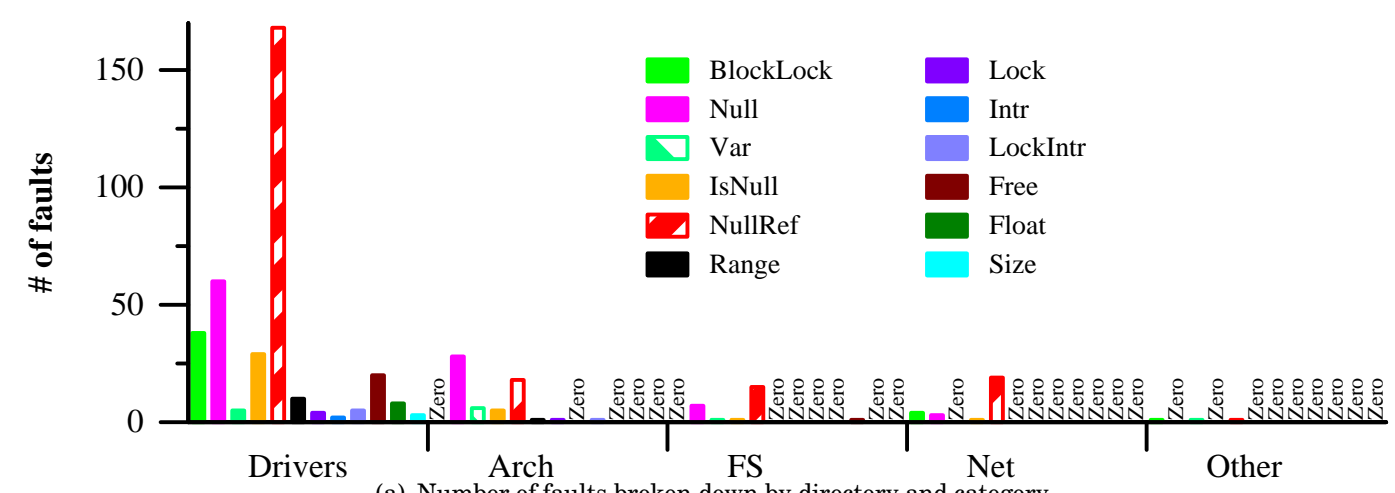

(a) Number of faults broken down by directory and category

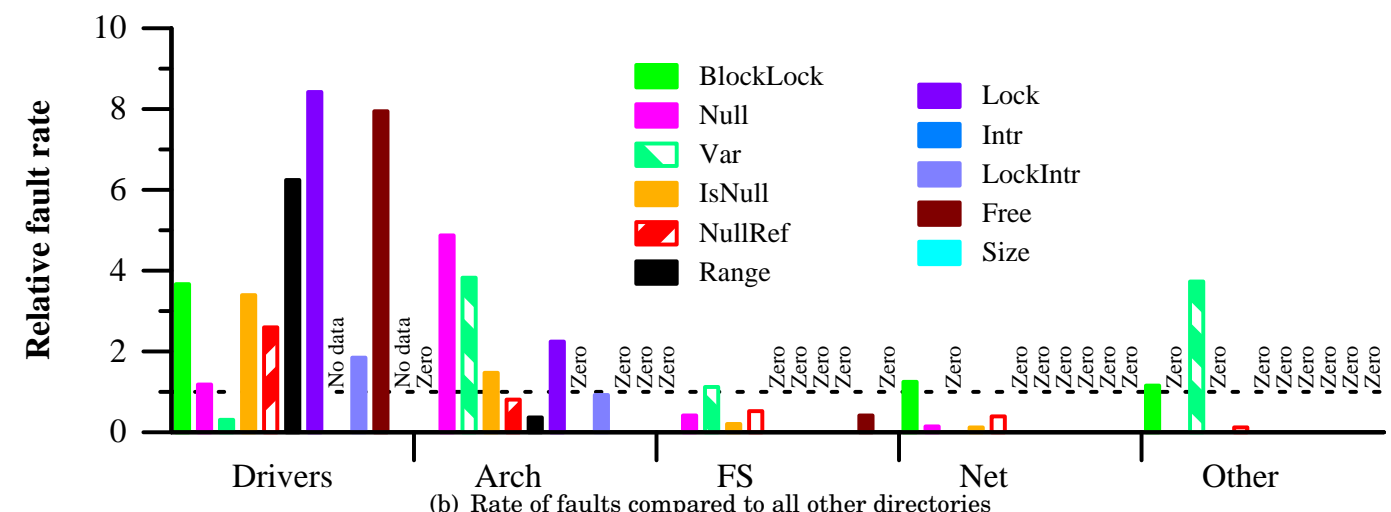

Fig. 5. Faults in Linux 2.4.1

\subsection{Where are the faults?}

In this section, the faults are classified using the same criterion as Chou et al., i.e., by subsystem and function size.

By subsystem. Chou et al. find that the largest number of faults is in the drivers directory and that the largest number of these faults are in the categories Block, Null, and Inull, with around 180, 95, and 50 faults in drivers, respectively 8 As shown in Figure 5(a), we also observe that the largest number of faults is in the drivers directory, with the largest number of these faults also being in BlockLock, Null, and Inull (IsNull and NullRef), although in different proportions.

A widely cited result of Chou et al. is that the drivers directory contains almost 7 times as many of a certain kind of faults (Lock) as all other directories combined. They computed this ratio using the following formula for each directory $d$, where $d$ refers to the directory $d$ itself and $\bar{d}$ refers to all of the code in all other directories:

$$
\text { fault rate }_{d} / \text { fault rate }_{\bar{d}}
$$

Figure 5(b) shows the values of the same formula, using our results. We obtain a similar ratio with a relative rate of over 8 for Lock in drivers, as compared to all other directories combined. We also find that the drivers directory has a rate of Free faults

\footnotetext{
${ }^{8}$ These numbers are approximated from the provided graphs.
} 
that is almost 8 times that of all other directories combined. Chou et al., however, found a fault rate of only around 1.75 times that of all other directories combined in this case. With both approaches, however, the absolute number of Free faults is rather small. Like Chou et al., we also observe a high fault rate in the arch directory for the Null checker, in both cases about 4.8 times that of all other directories combined. Finally, unlike Chou et al., we observe a high rate of Var faults in both arch and other. In the arch case, all of the Var faults found are for architectures other than x86. Indeed, overall for arch, we find 60 faults, but only 3 (all Null) in the x86 directory.

By function size. Figure 6 shows the percentage of faulty notes according to the size of the functions. Following Chou et al., we only consider functions with notes and divide these functions into 4 buckets, each containing the same number of functions. Like Chou et al., we find that the largest functions have the highest fault rate, as shown in Figure 6(a).

Linux, however, contains more small functions than large ones, as illustrated by Figure 7, which shows the distribution of the functions according to their size. Thus using same-sized buckets tends to group a wide range of larger functions into a single bucket. We thus decided to consider a logarithmic distribution of the functions into buckets, where the first bucket contains the smallest half of the functions, the next bucket contains the smallest half of the remaining functions, etc 9 Using this logarithmic bucketing strategy, Figure 6(b)] shows that it is actually the functions of the third bucket, having between 72 and 137 lines, that have the highest fault rate, at 1.05, although the largest functions continue to have a higher fault rate, at 0.77, than the smallest ones, at 0.49 .

To study the relation between function size and fault rate in more detail, Figure 6(c) increases the number of buckets to 6 , and considers all functions, not just those with notes. The curve with only the functions containing notes is given with a dotted line for reference. The same trend as found in Figure 6(b) is observed for small, mid-size, and large functions. This bucketing strategy, however, indicates the probability of a fault based solely on the function size, which represents a simpler criterion. As the two curves of Figure 6(c) are almost identical, the two strategies can be considered equivalent.

\subsection{How old are the faulty files?}

To explore the relation between the Linux 2.4.1 fault rate and file age, we first consider the distribution of files according to their age, as shown in Table IV] In Linux 2.4.1, there were few very recently added files; most of the files had been part of the Linux kernel for between 1 and 3 years. There is also an almost equal number of files for each of the numbers of years above 3. We thus decided to distribute the files equally among the buckets, as done by Chou et al., i.e. we first order the files according to their age and then assign each file to a bucket such that each bucket has almost the same number of files, as illustrated Figure 8. However, we again increase the number of buckets to 6 , to emphasize the relation between the faults and the file age for the youngest files, without disturbing the observation of other files, either young or old.

Table IV. File age distribution for Linux 2.4.1

\begin{tabular}{|l|c|c|c|c|c|c|c|c|}
\hline Age (in years) & 0 & 1 & 2 & 3 & 4 & 5 & 6 & 7 \\
\hline Number of files & 1330 & 1892 & 526 & 1217 & 541 & 627 & 325 & 322 \\
\hline
\end{tabular}

Chou et al. find that younger files have a higher fault rate, of up to $3 \%$ for the Null checker. We also find fault rates of more than $3 \%$ for the Null checker in 4 out of 6

$\overline{{ }^{9} \text { Note that }}$ at bucket boundaries, functions with the same length may appear in different buckets. 


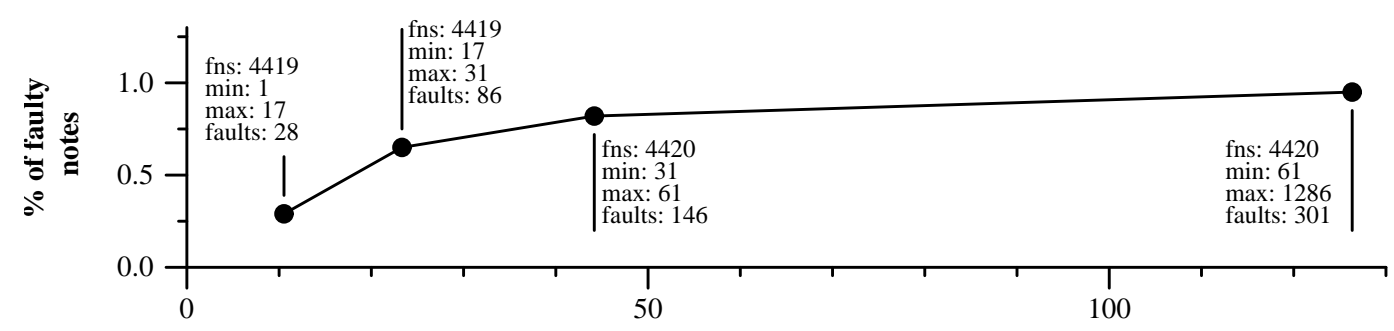

Function size in number of lines

(a) Functions with notes - 4 linear buckets (Chou et al.'s methodology)

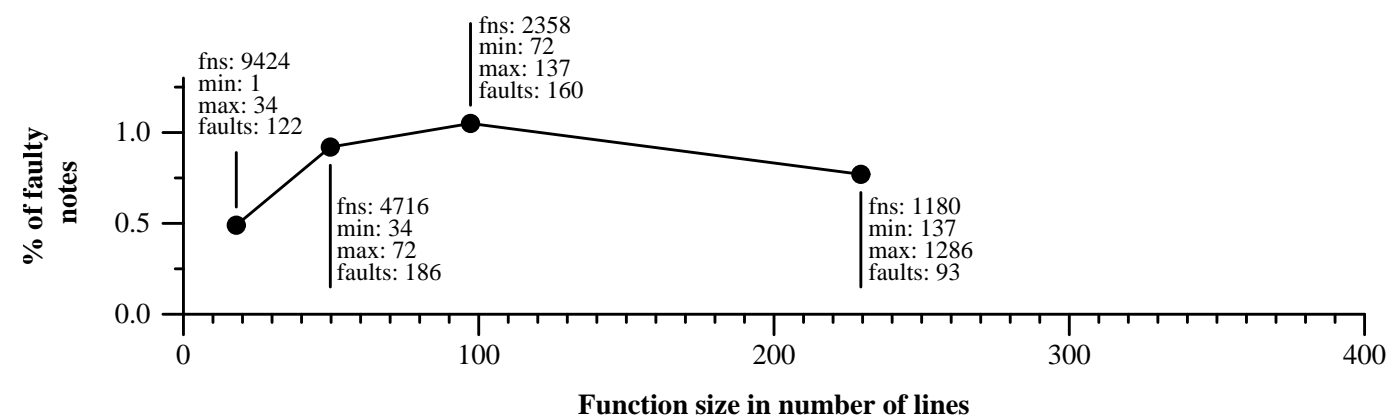

(b) Functions with notes - 4 logarithmic buckets

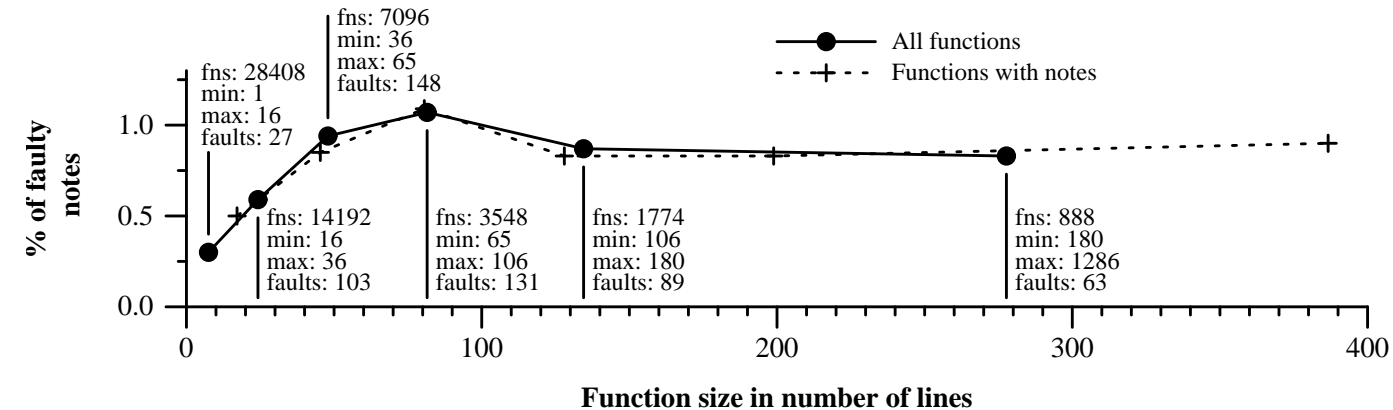

(c) All functions and functions with notes - 6 logarithmic buckets

At each point, fns is the number of functions affected to the bucket, $\mathbf{m i n}$, respectively $\mathbf{m a x}$ is the size of smallest, respectively largest, function of the bucket, and faults is the number of faults present in the functions assigned to the bucket.

Fig. 6. Fault rate by function size in 2.4.1

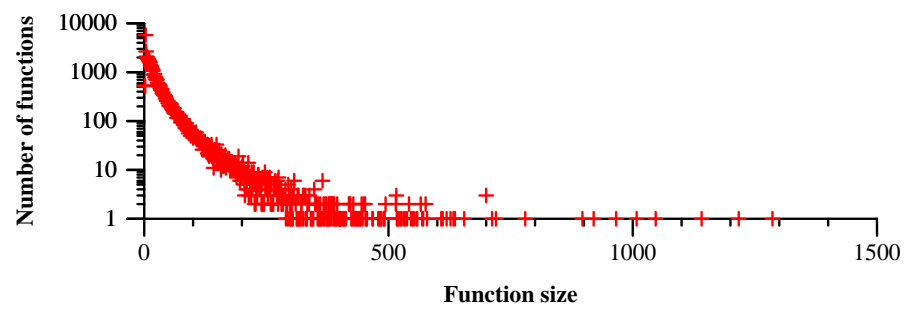

Fig. 7. Function size distribution 


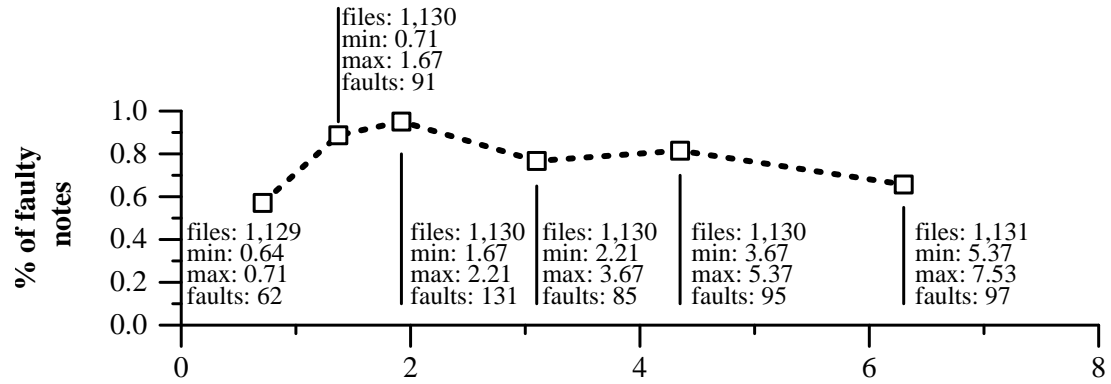

File age in years

At each point, files refers to the number of files considered to compute the point, min and max refer to the minimal or maximal age of the files in the bucket, and faults indicates the number of faults that occur in the bucket files.

Fig. 8. Fault rate by file age in 2.4 .1

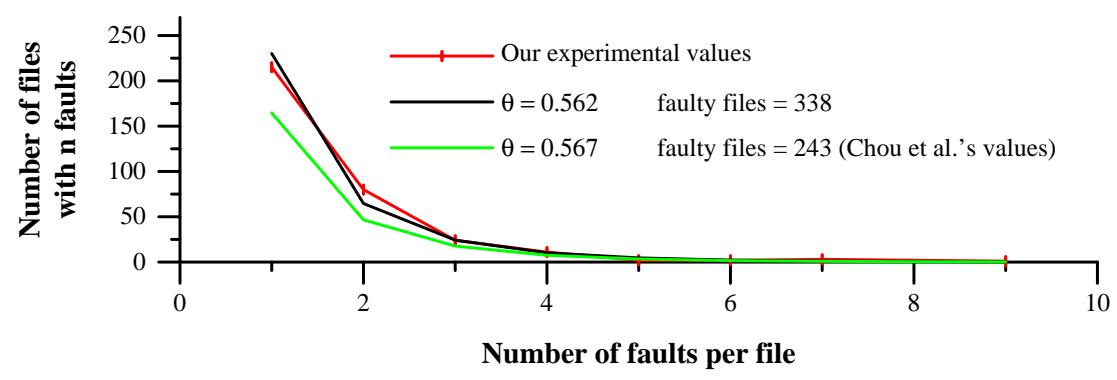

Fig. 9. Comparison of the error distributions

buckets, being the first three buckets and the last one. As illustrated by Figure 8 , moderately young files (between 8 months and 26 months) have the highest fault rates, over $0.89 \%$. However, the youngest files (under 8 months) have the lowest fault rate, $0.57 \%$. For files older than 2.5 years, we observe lower fault rates, above $0.82 \%$. Interestingly, middle aged files, of about 3 years, and the oldest files, of over 5 years have a lower fault rate than files between 4 and 5 years old.

In conclusion, our methodology gives comparable results with Chou et al. about the relation of faults and the age of files. However, by using two more buckets, we have been able to emphasize particularities of the newest and oldest files.

\subsection{How are faults distributed?}

Chou et al. plot the numbers of faults against the percentage of files containing each number of faults and find that for all of the checkers except Block, the resulting curve fits a log series distribution, as determined by $\chi^{2}$ test, with a $\theta$ value of 0.567 and a degree of confidence (p-value) of $0.79(79 \%)$. We observe a $\theta$ value of 0.562 and a $p$ value of 0.234 without Block. We conjecture that this low p-value is due to the fact that two files have 5 and 6 faults while three files have 7 faults each; such an increase for larger values is not compatible with a log series distribution. Nevertheless, the values involved are very small, and, as shown in Figure 9, the curve obtained from our experimental results fits well with the curve obtained using the corresponding calculated $\theta$ value. The curve obtained from Chou et al.'s value of $\theta$ is somewhat lower, because they found a smaller number of faulty files, probably due to having considered only the x86 architecture. 


\subsection{Assessment}

In this section, we have seen that our checkers do not find the same number of faults in Linux 2.4.1 code as the checkers of Chou et al. We recall that Chou et al.'s checkers are not very precisely described, and thus we expect that most of the differences are in how the checkers are defined. Furthermore, we do not consider exactly the same set of files. Nevertheless, the distribution of these faults among the various directories is roughly comparable, and their distribution among the files is also comparable. We thus conclude that our checkers are sufficient to provide a basis for comparison between Linux 2.6 and the previous versions studied by Chou et al.

\section{FAULTS IN LINUX 2.6}

We now assess the extent to which the trends observed for Linux 2.4.1 and previous versions continue to apply in Linux 2.6, and study the points of difficulty in kernel development today. We consider what has been the impact of the increasing code size and the addition of new features on code quality, and whether drivers are still a major problem.

Concretely, we study a period of over 7 years, beginning with the release of Linux 2.6.0 at the end of 2003 and ending in July 2011 with the release of Linux 3.0. For the complete set of Linux 2.6 kernels and for Linux 3.0, using the checkers described in Section 2.1, we obtain 51,449 reports, resulting in 5,169 reports after correlation, of which we have manually determined that 3,146 represent faults and the rest represent false positives.

\subsection{How many faults are there?}

We first analyze the relation between the code growth and the total number of faults in Linux 2.6. As shown in Figure 10(a), the number of the faults considered has held roughly steady over this period, with an overall increase of only $12.5 \%$. Indeed there was a decrease of $18 \%$ from 2.6.0 to the minimum value in the considered time period, which occured in 2.6.24. This latter decline is quite remarkable given that the code size increased by more than $50 \%$ during these 25 versions (Figure 11). However, since the minimum of 2.6.24, the number of faults has somewhat increased, including a jump of $17 \%$ between 2.6.28 and 2.6.30. As a result, we find in Linux $3.037 \%$ more faults than in 2.6.24, and $12.5 \%$ more than in 2.6.0. Still, due to the overall increase of $160 \%$ in code size from Linux 2.6.0 to Linux 3.0, the rate of faults per line of code has significantly decreased, by 57\%, as shown in Figure 10(b), and for version 3.0, we find fewer than 90 faults per $M L O C$. These observations are quite different from those for versions up through Linux 2.4.1: in those versions, there was a code size increase of over 17 times between Linux 1.0 and Linux 2.4.1 and an increase in the number of the faults considered of over 33 times [Chou et al. 2001]. Finally, Figure 10(c) reveals the reason for our observations about 2.6. Faults are still introduced, indeed at a growing rate, but in many versions even more faults are eliminated.

Figure 11 shows the number of each kind of fault found in Linux 2.6, separated for readability by their order of magnitude into those that have fewer than 100 faults at their maximum, shown in Figure 11(a), and the others, shown in Figure 11(b).

For many fault kinds, the number of faults is essentially constant over the considered period. Three notable exceptions are Lock in Linux 2.6.16 and 2.6.17, Null in Linux 2.6.29 and 2.6.34, and Float in Linux 2.6.30, as shown in Figure 11. In Linux 2.6.16, the functions mutex_lock and mutex_unlock were introduced to replace mutexlike occurrences of the semaphore functions down and up. 9 of the 11 Lock faults introduced in Linux 2.6.16 and 23 of the 25 Lock faults introduced in Linux 2.6.17 were in the use of mutex_lock. In Linux 2.6.29, the btrfs file system was introduced, as 


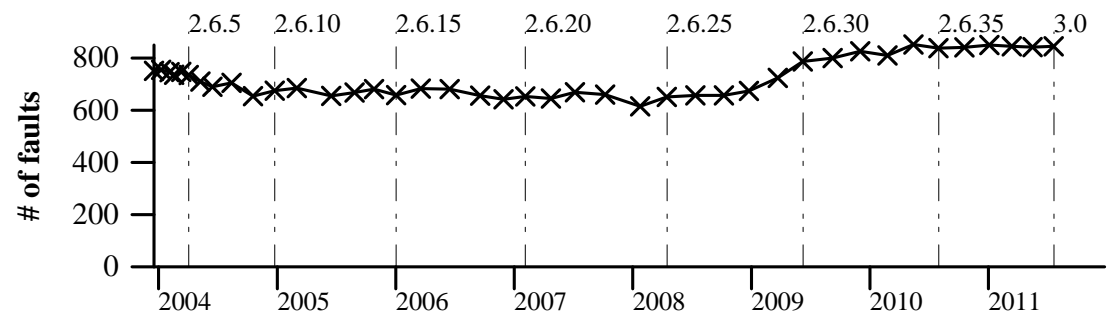

(a) Faults

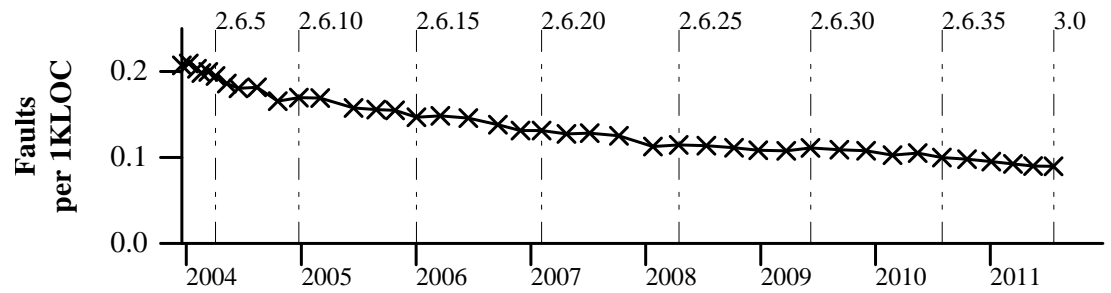

(b) Fault density (Faults per 1KLOC)

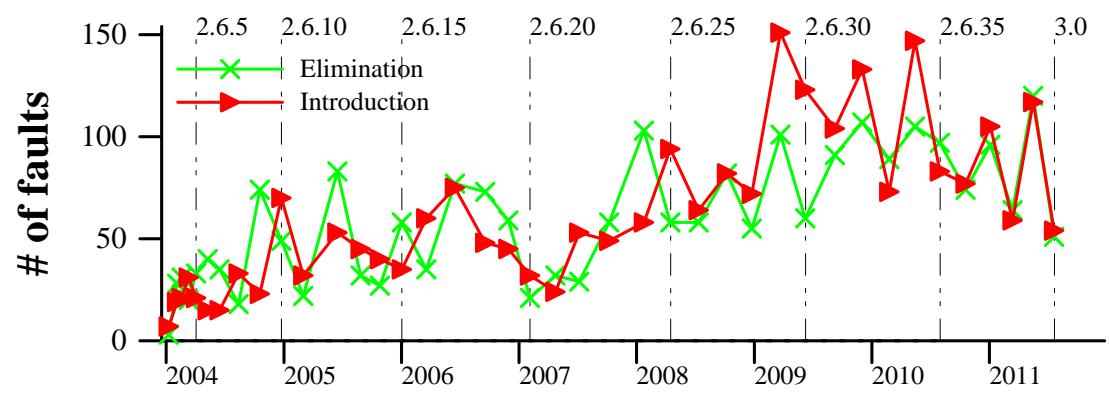

(c) Introduction and elimination of faults

Fig. 10. Faults in Linux 2.6.0 to 3.0

seen in Figure 3. 31 Null faults were added with this code. 7 more Null faults were added in drivers/staging, which more than tripled in size at this time. 31 other Null faults were also added in this version. Next, in Linux 2.6.30 there was a substantial increase in the number of Comedi drivers [Comedi 1999] in drivers/staging. All of the 21 Float faults introduced in this version were in two Comedi files. These faults are still present in Linux 3.0. Recall, however, that staging drivers are not included in Linux distributions. Finally, in Linux 2.6.34, 75 Null faults, out of 119, were introduced in the arch and fs directories.

In the IsNull category, the number of faults decreases across the studied period. Nevertheless two spikes in the last two years can be observed. Indeed, 22 faults were introduced between 2.6.28 and 2.6.34, of which 7 were introduced in 2.6.29. These faults were introduced almost entirely in drivers. In Linux 2.6.35, the number of faults dropped to only 8, but it quickly grew up to 19 faults in 3.0 through the introduction of 18 new faults (note that some faults were corrected at the same time, implying some fault turnover). In each version, the faults were introduced mainly in 


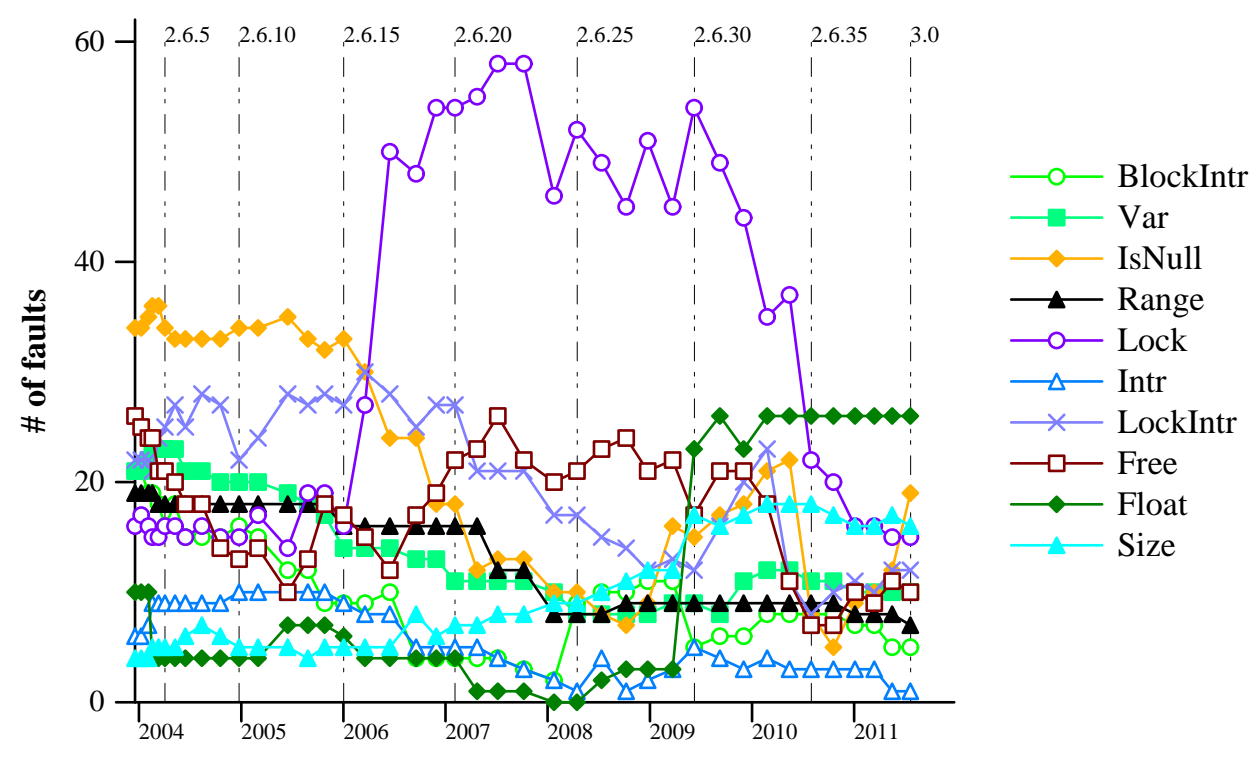

(a) Fewer than 100 faults

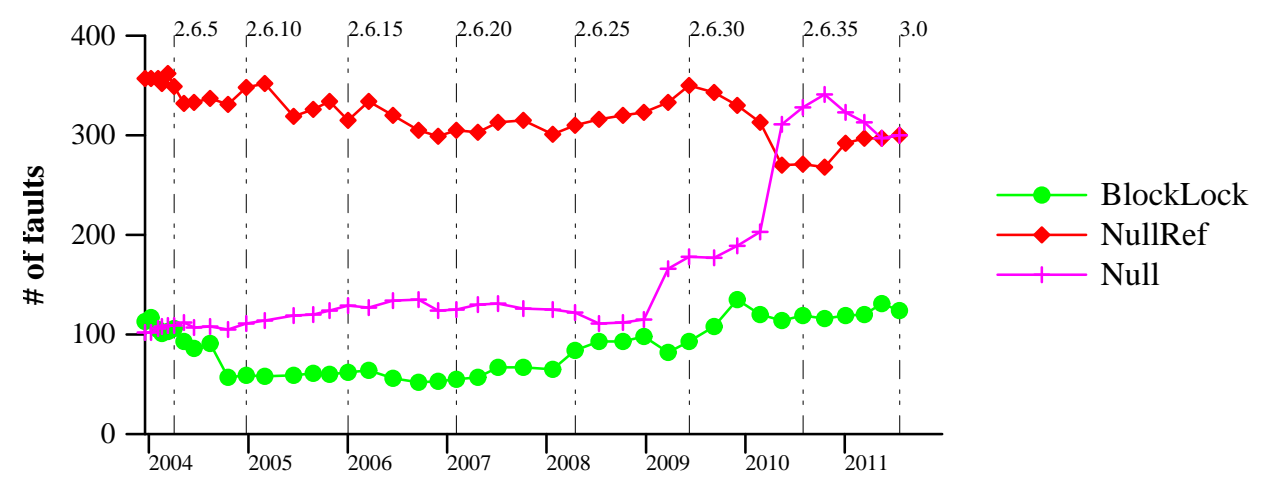

(b) More than 100 faults (BlockLock, NullRef and Null)

Fig. 11. Faults through time

drivers. So, between 2.6.28 and 2.6.34, 40 IsNull faults were introduced, of which 10 were in the staging directory and 32 in other drivers.

As shown in Figure 12, the fault rate, i.e., the ratio of observed faults to the number of notes, for the considered fault kinds confirms the increase in reliability (Float is omitted, as described in Section(2.2). As the number of notes increases roughly with the size of the Linux kernel while the number of faults is relatively stable, the fault rate tends to decline. The main increases, in Lock and Null, are due to the introduction of mutex_lock and the btrfs file system, respectively, as mentioned previously.

\subsection{Where are the faults?}

The presence of a high rate of faults in a certain kind of code may indicate that this kind of code overall needs more attention. Indeed, Chou et al.'s work motivated stud- 

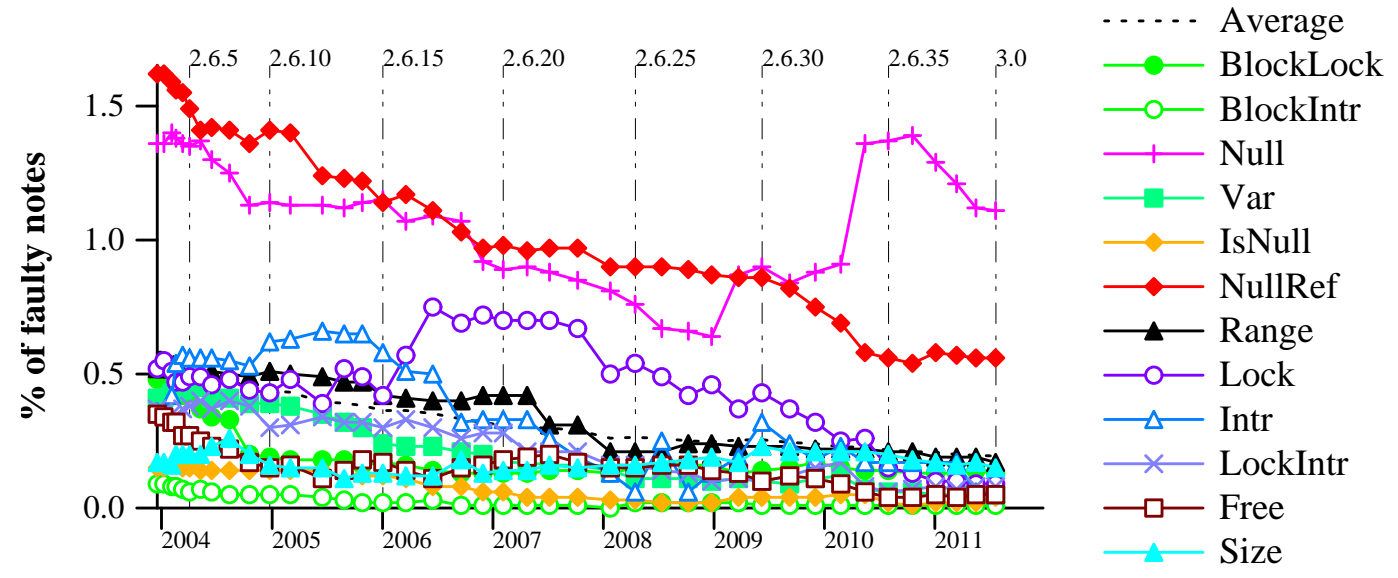

Fig. 12. Fault rate per fault kind

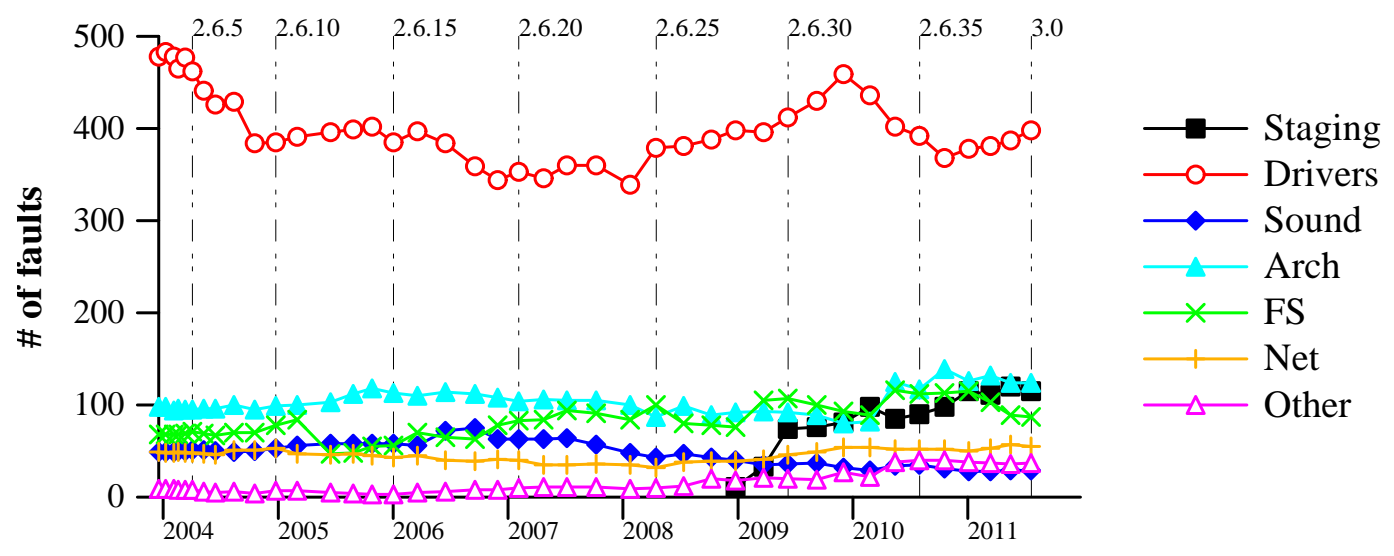

Fig. 13. Faults per directory

ies of many kinds of driver faults, going beyond the fault kinds they considered. Many properties of the Linux kernel have, however, changed since 2001, and so we reinvestigate what kind of code has the highest rate of faults, to determine whether attention should now be placed elsewhere.

As shown in Figure 13 the largest number of faults is still in drivers, which indeed makes up over half of the Linux kernel source code. The second-largest number of faults is in arch, accompanied by $\mathrm{fs}$ and drivers/staging in recent versions. In contrast to the case of Linux 2.4.1, however, as shown in Figure 14 drivers no longer has the largest fault rate, and indeed since Linux 2.6.19 its fault rate has been right at the average. There was not a large increase in the number of drivers notes at that time, so this decrease is indicative of the amount of attention drivers receive in the peer reviewing process. Arch on the other hand has many faults and relatively little code, and so it has the highest fault rate throughout most of Linux 2.6. Around 30\% of the arch faults are Null faults, although there appears to be no pattern to their introduction. Over $90 \%$ of the arch faults are outside of the x86/i386 directories, with many of these faults being in the ppc and powerpc code. 


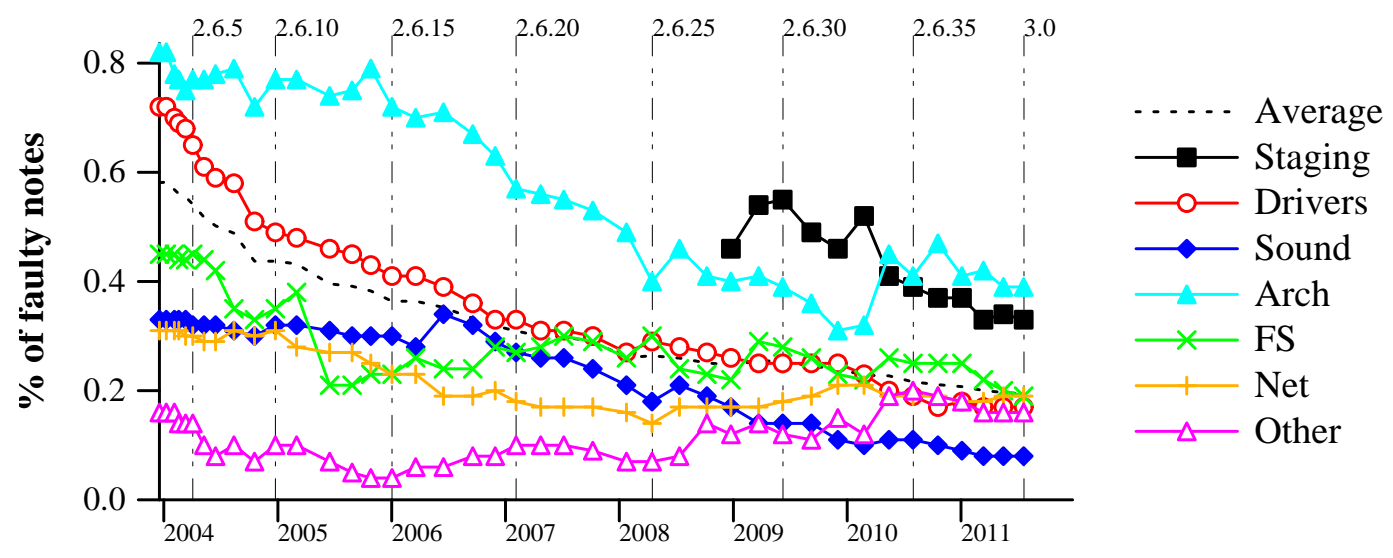

Fig. 14. Fault rate per directory

The largest numbers of faults in $f s$ are in cifs, ocfs 2 and btrfs. For the cifs filesystem, there are over 40 faults in Linux 2.6.0. But the number of faults suddenly drops from 52 to 16 between 2.6.11 and 2.6.12 and only two such faults remain in 3.0. For the ocfs2 filesystem, there are 10-14 faults per version starting in Linux 2.6.16 until the versions 2.6.39 and 3.0, where there are respectively only 4 and 5 faults remaining. For the btrfs filesystem, there are respectively 36 and 38 faults in Linux 2.6.29 and 2.6.30. Again, there are respectively only 8 and 6 faults in the two last versions. All of these faults are in recently introduced file systems: cifs was introduced in Linux 2.5.42, ocfs2 in Linux 2.6.16, and btrfs in Linux 2.6.29.

Drivers/staging, introduced in Linux 2.6.28, also has a high fault rate, exceeding that of arch. This directory is thus receiving drivers that are not yet mature, as intended. The introduction of drivers/staging, however, has no impact on the fault rate of drivers, as drivers/staging accommodates drivers that would not otherwise be accepted into the Linux kernel source tree. Such drivers benefit from the expertise of the Linux maintainers, and are updated according to API changes with the rest of the kernel.

For Linux 2.4.1, we observed that drivers had a much higher fault rate for certain kinds of faults than other directories. Figure 15 shows the comparison of fault rates for Linux 3.0. It is more common that drivers/staging, arch, or other has the highest fault rate, indicating that the drivers that are intended for use in the Linux kernel are no longer the main source of faults. Indeed, there are about 11 times more faulty notes in drivers/staging than in all other directories. The maximum for arch and other is respectively 9 for BlockIntr, and 10 for LockIntr. In comparison, drivers has 2.3 more faulty BlockLock notes than all other directories in Linux 3.0. This worst case of the drivers directory is more than 4 times smaller than the three other maximum values mentioned above.

For staging, dereference of a NULL pointer (IsNULL) and missing memory frees (Free) are the most common errors, with respectively 8 and 11 times more errors in staging than in the rest of the kernel. For arch, the highest ratio is for BlockIntr which is 9 times higher than in the rest of the kernel. Arch has also high ratios for Null, Var and LockIntr, with a factor of 4 in these cases. In the case of $\mathrm{fs}$, the most problematic point is Lock with a ratio of 3.2. Sound and net have the smallest fault rates. In a preliminary version of this work, we compared fault rates between directories in Linux 2.6.33 (Figure 11 of [Palix et al. 2011]). The two highest rates for sound 


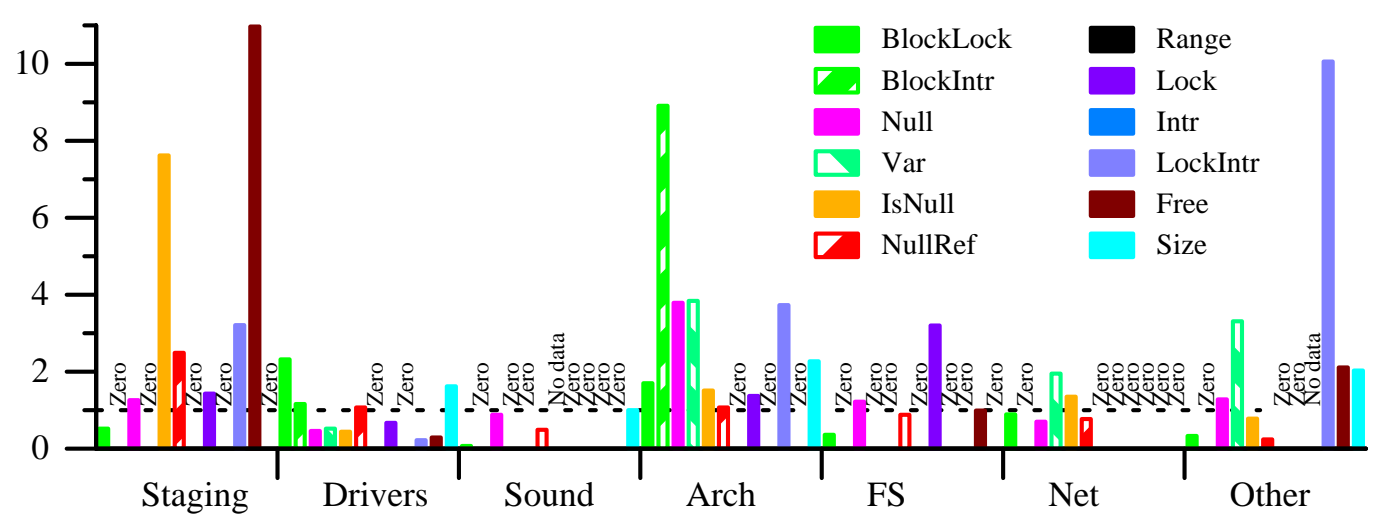

Fig. 15. Fault rates compared to all other directories (Linux 3.0)

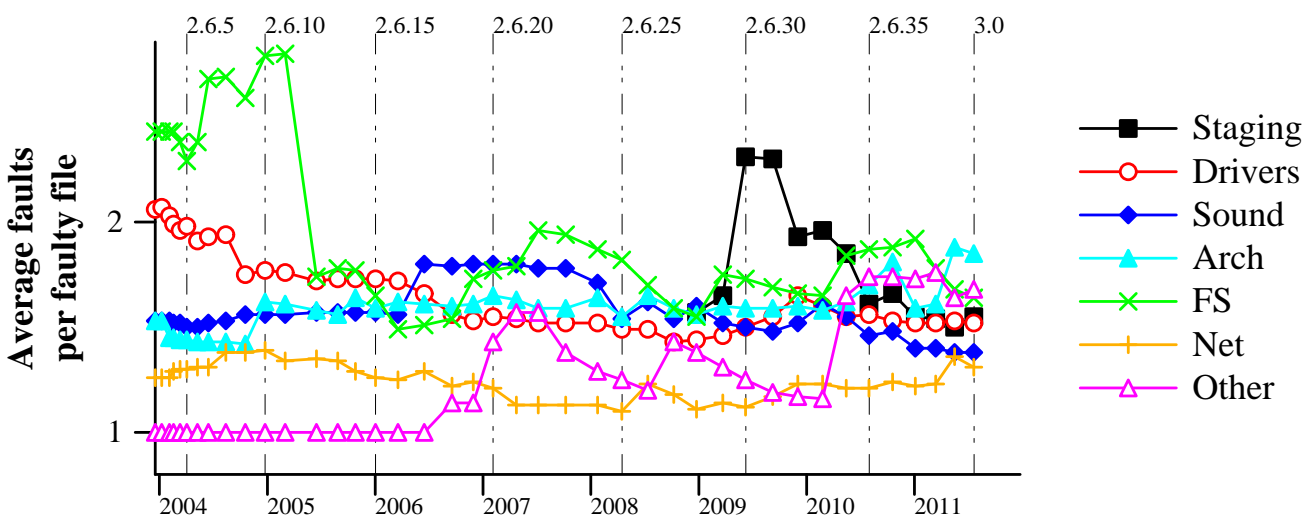

Fig. 16. Faults per faulty file per directory

found in that study are no longer visible in the results for Linux 3.0, indicating the sound has improved compared to the other directories.

Finally, in Figure 16, we consider the number of faults per file containing at least one fault. The highest average number of faults per faulty file is for $f \mathrm{~s}$ in the versions prior to 2.6.12, at 2.8 faults. In this case, there was a single file with many NullRef faults, as many as 45 in Linux 2.6.11. In later versions, the highest average is for drivers/staging, for which the average was over 2 in Linux 2.6.30. At that point, a large number of drivers had recently been introduced in this directory. Many of these faults have been corrected and the rate of entry of new drivers has slowed, and thus the average has dropped to around 1.5, close to that of other directories. Sound had a relatively high number of faults per faulty file starting in Linux 2.6.16 with the introduction of mutex_lock; faulty functions often contain more than one mutex_lock, and thus a single omitted mutex_unlock may result in multiple Lock reports.

\subsection{How long do faults live?}

Eliminating a fault in Linux code is a three step process. First, the fault must be detected, either manually or using a tool. Then, it must be corrected, and a patch submitted to the appropriate maintainers. Then, the patch must be accepted by a hierarchy of maintainers, ending with Linus Torvalds. Finally, there is a delay of up to 3 months until the next release. It has thus been found that most patches are integrated within 
3-6 months [Jiang et al. 2013] into a Linux release, i.e. it takes one or two releases to integrate patches. The lifespan of a fault, modulo this three/six-month delay, is an indication of the efficiency of the fault-elimination process.

Fault survival analysis. A challenge in measuring the lifetime of faults present within a time period is that some faults may have been introduced before the time period began, while others may last after the time period ends. In this section, we consider the probability that a fault, that is observed at least once between Linux 2.6.0 and Linux 3.0, reaches a certain age. A fault that was introduced before the considered time period (i.e. before Linux 2.6.0) is said to have its lifetime left censored, while a fault that persists after the considered time period (i.e. after Linux 3.0) is said to have its lifetime right censored. The problem of censoring is addressed by a survival analysis with the Kaplan-Meier estimator [Kaplan and Meier 1958]. Survival analysis gives the probability that an observation (e.g., the death age of faults) reaches a given value. The Kaplan-Meier estimator corrects the bias introduced by the partial observations (i.e., the observations of faults where only a lower bound of the age is known because either the fault introduction or the fault removal was unobserved), making the computed average lifespan unbiased as compared to the average lifespan of the raw observations. To refine the estimator, the approach allows approximating partial observations by an interval. For complete observations, the exact value of the fault age at death is used.

To handle the censored data of Linux 2.6.0, as we did not know the exact lifespan of the faults, we bound the lifespan by an interval and thus set a lower-bound and an upper-bound on the lifespan of the Linux 2.6.0 faults. The lower-bound is always the observed lifespan, i.e., we assume that the fault was introduced where we first observe it, in Linux 2.6.0. For the upper-bound, we considered three alternatives: 1) minimize the upper bound by setting it equal to the lower-bound (all Linux 2.6.0 faults are new), 2) maximize the upper bound by extending the lifespan up to Linux 1.0 and 3) set the upper bound by extending the lifespan up to the median point between Linux 1.0 and Linux 2.6.0, which is in January 1999, near the release of Linux 2.2.0.

Figure [17 reports the survival analysis of faults, computed with $\mathrm{R}$ [R Core Team 2014a], according to four configurations: first, ignoring the faults of Linux 2.6.0 and then each of the three alternatives mentioned above. The first configuration, illustrated in black at the bottom left, only considers the faults introduced after Linux 2.6.0, as they are at worst only right-censored. The second one, the solid green line in the middle, considers that all Linux 2.6.0 faults are new, and thus gives the lower bound of 0 as the estimation. The third one, in red at the top, considers that the Linux 2.6.0 faults may have been introduced in Linux 1.0, and thus gives the upper bound of the estimation. Finally, the fourth one, in dotted black in the middle, gives a median estimation where the Linux 2.6.0 faults may have been introduced at any time between January 1999 and December 2003 (Linux 2.6.0). This may extend the lifespan of the faults of Linux 2.6 .0 by up to five years with respect to the observed lifespan.

The faults considered in the first configuration have an average lifespan of 2.3 years \pm 22 days, and a median lifespan of 1.3 years. As shown in Figure 17, this is the lowest lifespan observed. The faults, for which the birth is observed, are thus fixed quicker than the set of all the faults, i.e., the set that includes the faults already present in Linux 2.6.0 (as considered in the remaining configurations). For the second and third configurations, respectively the min and max strategies, the average lifespan ranges from 2.4 to 3.7 years, and the median lifespan from 1.5 to 2.2 years. The strategy for left-censoring thus has an impact of up to about 1 year. For the median estimation (fourth configuration), the average lifespan of a fault is 2.9 years \pm 19 days, and the 


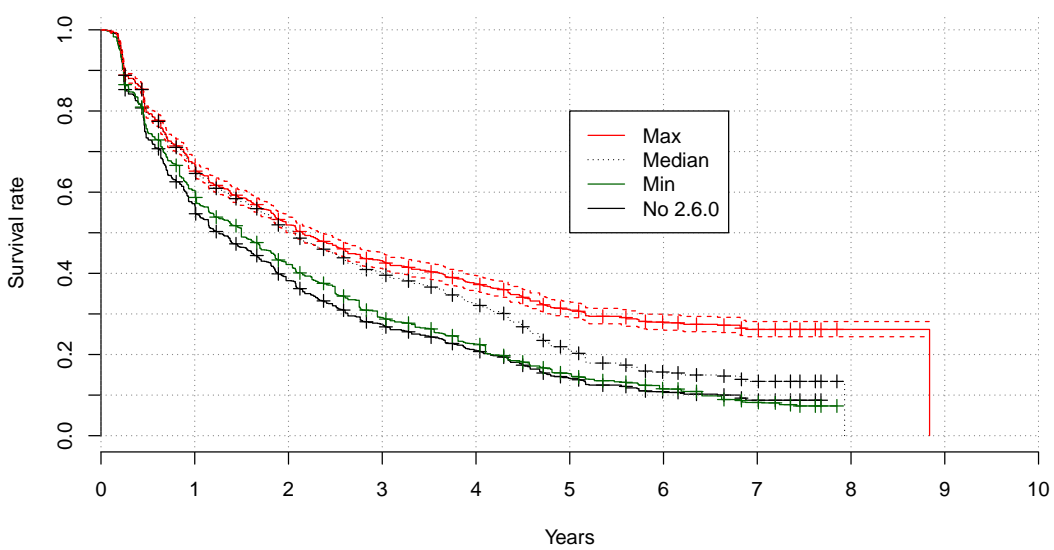

Fig. 17. Kaplan-Meier survival analysis of a fault

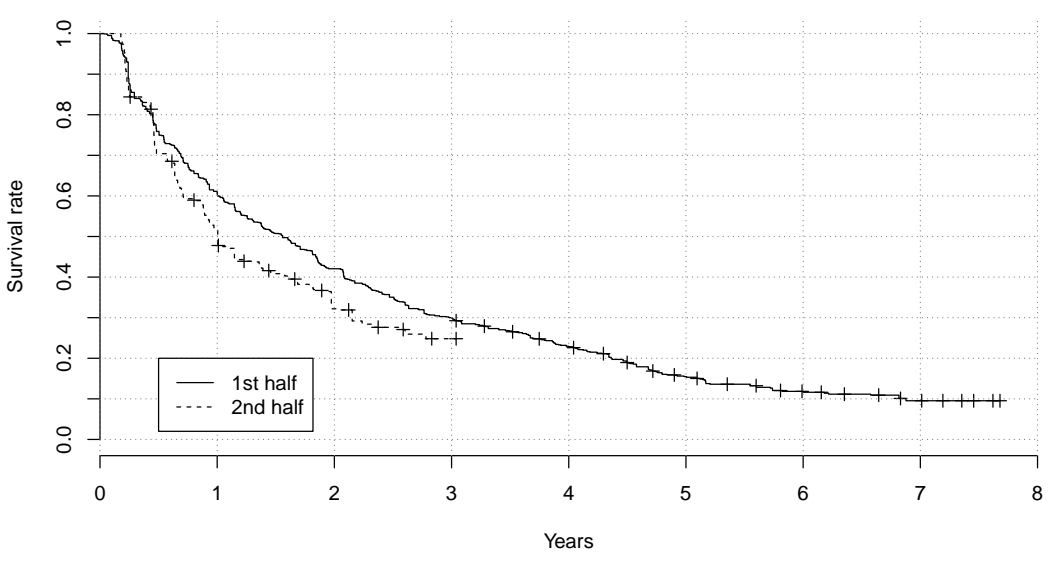

Fig. 18. Kaplan-Meier survival analysis comparison

median lifespan is 2.1 years. This strategy affects the average lifespan more than the median lifespan. We can thus conclude that the average lifespan is about between 2.5 and 3.0 years and the median lifespan is about 2 years.

Figure 18 compares the lifespans of faults introduced in the first half of the versions (with the first configuration, i.e. ignoring the Linux 2.6.0 faults) with the lifespans of faults introduced in the last half of the versions. We observe an average lifespan of 2.41 years and 2.60 years, for respectively the first and the last half of the versions. These results show that the average lifespan has increased. However, the median lifespan has decreased from 1.56 to 1.00 years.

In Figures 17] and [18, each cross corresponds to a right-censored fault. The 0.95 confidence band is given as a pair of dotted lines in Figure 17, for the upper-bound estimation. This band is rather tight and is similar for all of the curves (not shown for the sake of clarity).

Lifespan per directory and fault kind. Figure 19 presents the average lifespan of faults across Linux 2.6, by directory and by fault kind. We omit drivers/staging because it was only introduced recently. The lifespan averages are reported by the Kaplan-Meier estimator with the median strategy. 


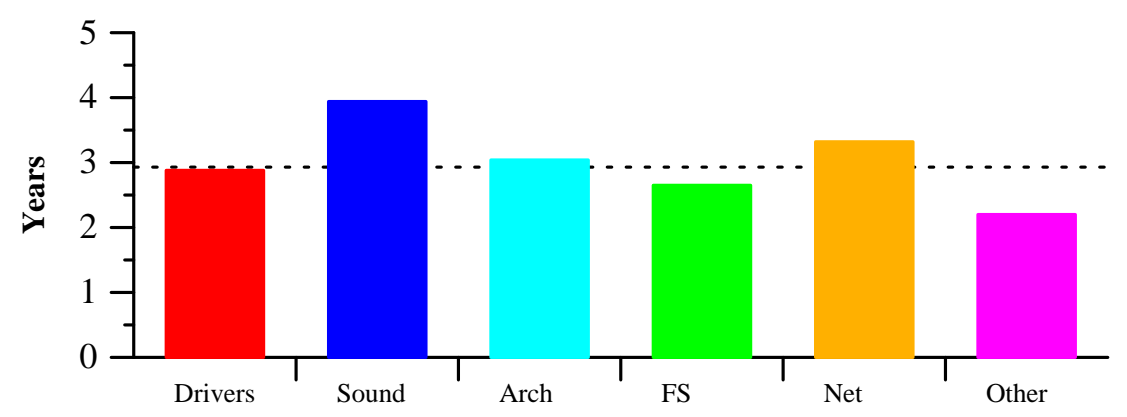

(a) Per directory

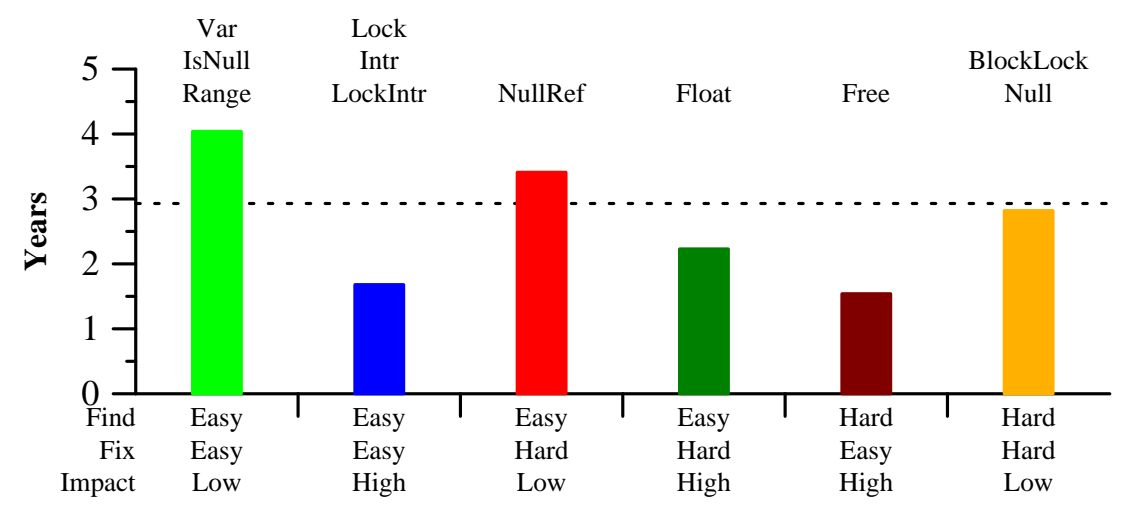

(b) Per finding and fixing difficulty, and impact likelihood

Fig. 19. Average fault lifespans (without staging)

The average fault lifespan across all files of Linux 2.6 is 2.93 years, as indicated by the horizontal dotted line in Figure 19] The lifespans vary somewhat by directory. As shown in Figure 19(a), the average lifespan of faults in the drivers directory is the same as the average lifespan of all faults, and indeed is less than the average lifespan of faults in the sound directory. Sound faults now have the longest average lifespan. Sound used to be part of drivers; it may be that the sound drivers are no longer benefiting from the attention that other drivers receive. In Figure 14 of Section 5.2 (page 20), we showed that the fault rates of $f s$ and arch are now worse than that of drivers. Here, we find similarly that faults in arch have a longer lifespan than faults in drivers. However, the faults in fs have the second smallest lifespan.

For the fault kinds, Figure 19(b) shows that the average lifespans correspond roughly to our assessment of the difficulty of finding and fixing the faults and their likelihood of impact (Table I). In particular, all of the fault kinds we have designated as having high impact, meaning that the fault is likely to have an observable effect if the containing function is executed, have the lowest average lifespans. Moreover, among the high impact faults, the ones that are easily fixed have the lowest average lifespans. On the other hand, the ease of finding the faults has little impact on their lifespan, showing that developers are willing to invest in tracking down any faults that cause obvious problems, and are prone to quickly accept simple fixes. 


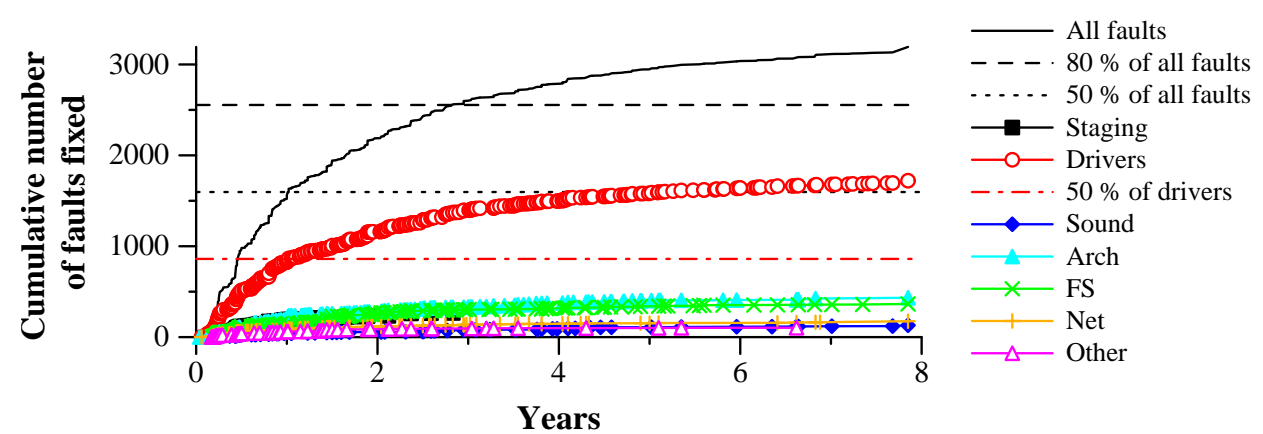

(a) Cumulative number

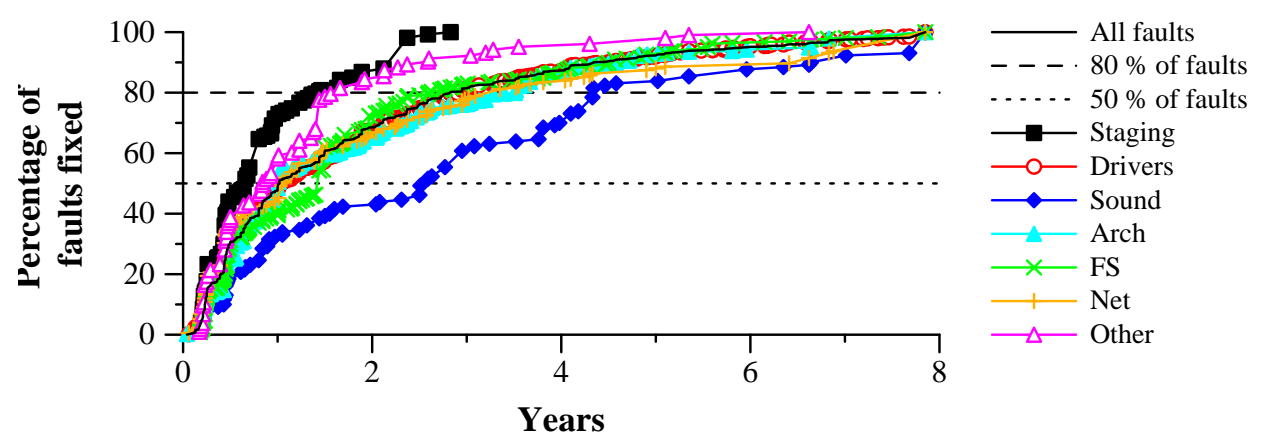

(b) Percentage

Fig. 20. Fixed faults per amount of elapsed time

Figure 20 examines fault lifespans in more detail, by showing the number of faults (Figure 20(a)) and the percentage of faults (Figure 20(b)) that have been fixed in less than each amount of time. In these figures, we include the faults already present in Linux 2.6.0 and the faults still remaining in Linux 3.0. Staging is also added back to compare it with the other directories, but as it was introduced only two and a half years before 3.0, no staging fault can have a lifetime longer than 2.5 years. If we consider all of the faults, half of them were fixed within just above one year, but it took about 3 years to fix $80 \%$ of them. This global trend is observed for almost all of the directories. Indeed, half of the faults were fixed in around one year for each subsystem, except sound where 2.5 years were required to fix half of the faults. In 2010 , we did a similar study, reported in a preliminary version of this paper [Palix et al. 2011], and found for staging that half of the faults were fixed within 6 months. We now observe that the time is almost 8 months. These drivers may thus have lost the attention of code reviewers as compared to the time at which staging was created.

Origin of faults. Figure 21 shows the lifetime of each of the faults found in our study 10 The faults are ordered by birth date, with faults of Linux 2.6.0 at the bottom of the graph and new faults of Linux 3.0 at the top. For each version, the faults are further ordered by their death age. The 751 faults in Linux 2.6.0 are marked as introduced at that point, even though they may have been introduced earlier. Of the faults introduced and eliminated within the period considered, $35 \%$ of the faults introduced in or after Linux 2.6.0 were introduced with the file and $12 \%$ of the faults eliminated

${ }^{10}$ The details of this figure may be more apparent on a printout than on a monitor. 
before Linux 3.0 were eliminated with the file. These percentages have remained stable as compared to the ones of our previous study [Palix et al. 2011].

While we have seen that the total number of faults is essentially constant across the versions, Figure 22 shows that since Linux 2.6.27, a significantly larger number of faults have been introduced. Null and NullRef faults predominate, with for example $51 \%$ of the added faults in Linux 2.6.27 being NullRef faults, most of which were introduced in various drivers. In Linux 2.6.30 and Linux 2.6.32, $43 \%$ and $26 \%$ of the introduced faults, respectively, were in drivers/staging. In each case, about half of the introduced faults were fixed within a few versions.

Figure 22 shows the number of faults in each version (highest point of each line) that are also present in each of the previous and successive versions. For instance about 200 faults of Linux 2.6.0 are still present in Linux 2.6.23, as seen by following the Linux 2.6.23 curve backward until Linux 2.6.0 was released. Linux 2.6.23 furthermore has more than 600 faults, and about 200 of these faults remain until Linux 3.0, as seen by following the curve forward up to the Linux 3.0 release. Except for the increase at Linux 2.6.29 and 2.6.30, as previously noted, the height of all of the lines is fairly similar, indicating that the rate of introductions and eliminations of faults across the versions is relatively stable. These facts indicate a maturity in the Linux code and its development model. However, the angle of the lines is becoming sharper indicating the improvement of fault correction, as previously reported in Figure 18.

Use of tools. In principle, since the work of Chou et al., it has been possible to find all of the considered faults using tools. Figure 23 shows the number of patches in each Linux version that mention one of the fault-finding tools Coccinelle [Padioleau et al. 2008] (used in this paper), Coverity [Coverity 2008] (the commercial version of Chou et al.'s xgcc tool), Sparse [Searls 2004; Sparse 2003], and Smatch [The Kernel Janitors 2010]. As developers are not obliged to mention the tools they use, these results may be an underestimation. However, we observe a Pearson's correlation coefficient of 0.641 between the number of tool-based patches and the elimination of faults, which are reported respectively in Figure 23 and Figure 10(c).

The use of the various tools is somewhat variable across the different versions. In the case of Coverity, the main use occurred between 2006 and 2009, when its application to open-source software, including Linux, was funded by the US Department of Homeland Security [CDHSE 2009]. Sparse usage shows several large peaks, at 2.6.25 and at 2.6.29-30, and smaller ones at 2.6.35, 2.6.37 and 2.6.39. For 2.6.25, 32 developers used Sparse to produce 129 patches, but half of these patches were produced by only 3 developers. For versions 2.6.29 and 2.6.30, a single developer was responsible for 140 of the 253 Sparse-related patches. This developer fixed faults across the entire kernel. Each of the three small peaks comes from the use of Sparse by a single developer, who produced between 10 and 25 patches each time, among about 20 other developers. This developer was different for each peak. The tools have also been used to find a wider range of faults than those considered in this paper. For example, in Linux 2.6.24, only about half of the patches that mention Coverity relate to the kinds of faults we consider, particularly Null, IsNull, NullRef, and Free. Overall, despite the variability in usage, the results show a willingness on the part of the Linux developers to use fault-finding tools and to pay attention to the kinds of faults that they find.

Around the time of our preliminary study of faults in Linux 2.6 [Palix et al. 2011], we contributed a number of patches using Coccinelle based on our findings. These include patches on faults found in released versions, but also faults introduced between versions, as found using the head of the Linux git repository. While these patches made it possible to validate our results, it also meant that our own patches may interfere with the results on each version reported here. During the period of Linux 2.6.30 and 


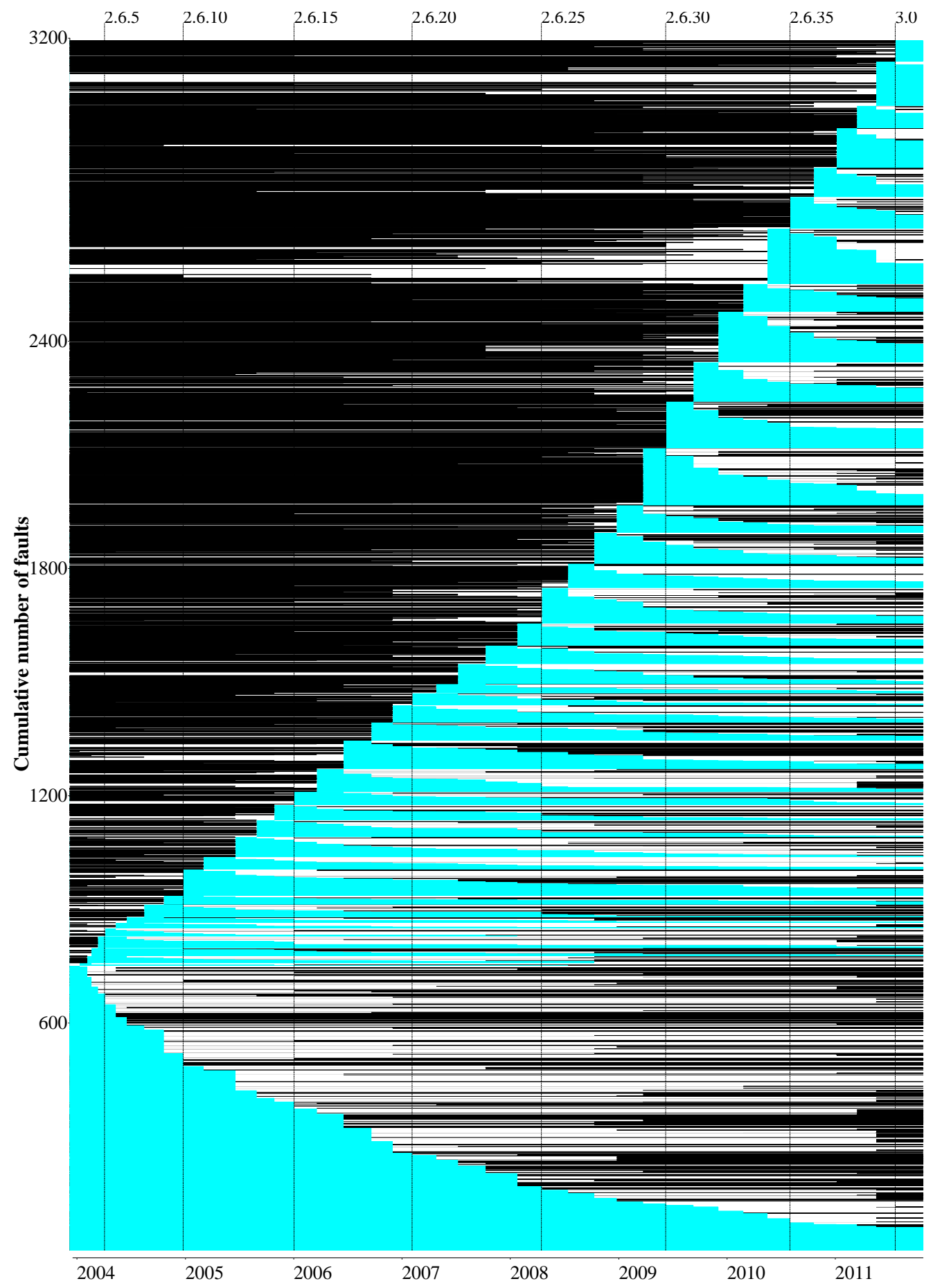

Fig. 21. Lifetime of faults. Each row represents a separate fault. Blue (grey) lines indicate the periods where the fault is present. Black lines indicate the period where the file containing the fault does not exist and white lines indicate the period where the file does exist but the fault does not. 


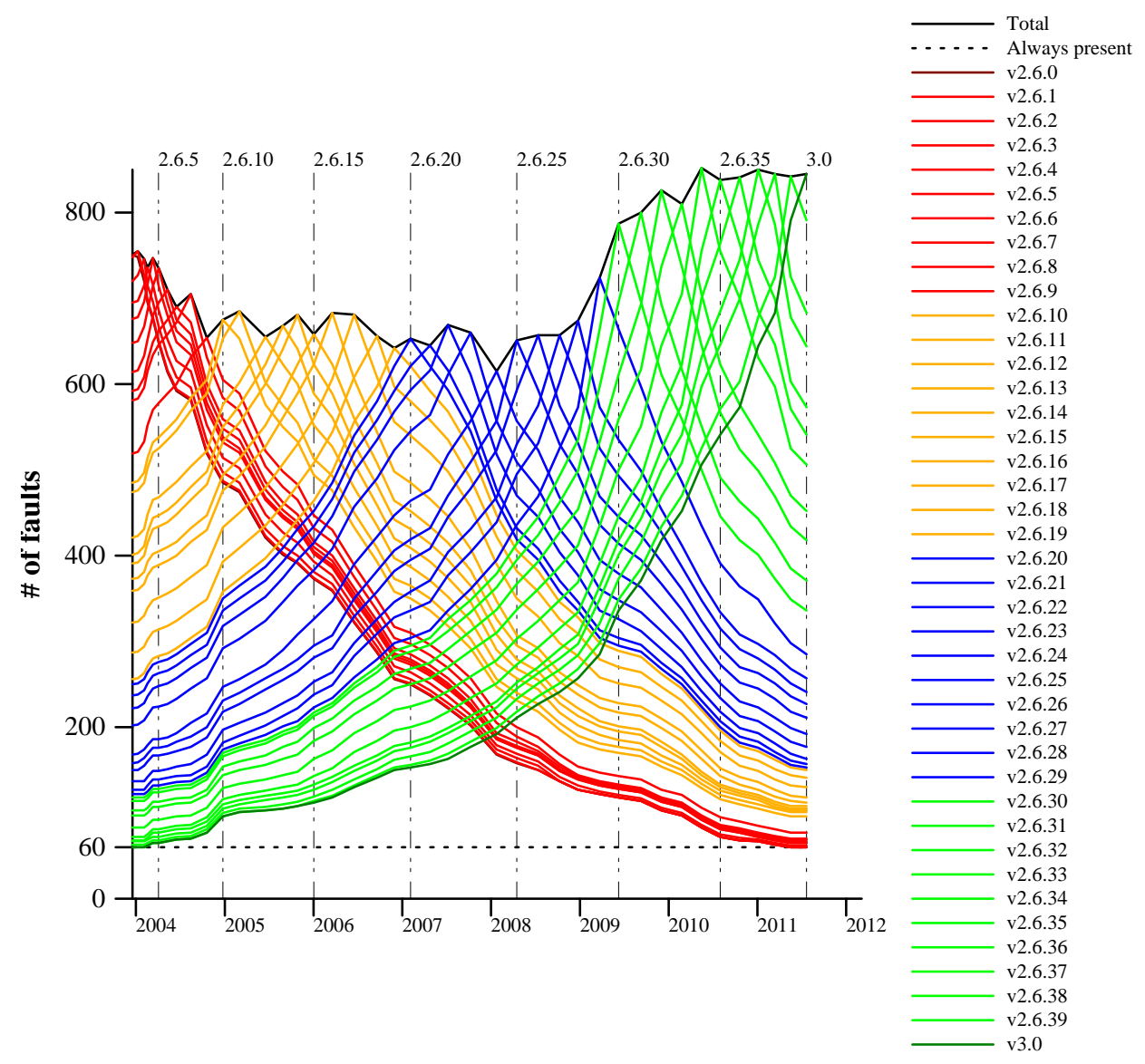

Fig. 22. Lifetime of faults across versions

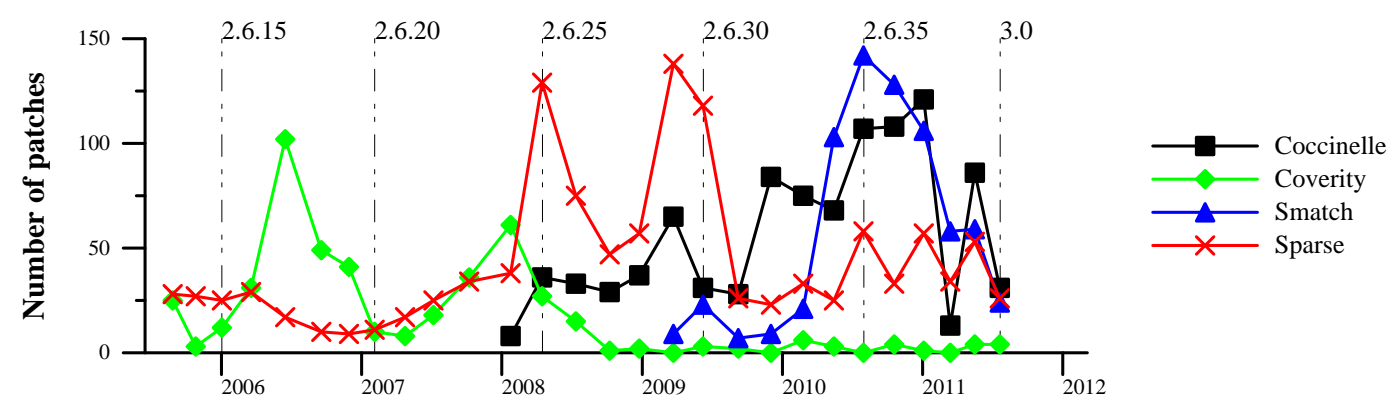

Fig. 23. Tool usage since the introduction of git (Linux 2.6.12) 
Linux 3.0, which contains the largest number of uses of Coccinelle, we find that just under $22 \%$ of the patches that refer to Coccinelle, by ourselves or others, fix the kinds of faults considered in this paper. Most of these faults are related to NULL pointers. In contrast, the patches submitted using Coccinelle typically address memory leaks and modernizing various APIs, which are not considered here. Thus, we consider that our contributions to the Linux kernel have only had a small impact on the results presented in this paper.

\section{CODE QUALITY PREDICTABILITY IN LINUX 2.6}

In the software-engineering community, substantial work has been done on identifying metrics that predict code quality [Munson and Elbaum 1998; Nagappan and Ball 2005; Bird et al. 2009]. Among these, we consider human-related factors and how the code is structured and evolves.

\subsection{Human factors}

We first evaluate the developer activity in the Linux project, and then how developer commitment is related to the introduction of faults.

Developer activity. In the Linux development model, anyone (an author) can submit a patch to a maintainer and to relevant mailing lists for public review. If the patch is accepted, it is then picked up by a committer (often the maintainer), who commits it into his git repository. The committer's git repository is then propagated to Linus Torvalds, who makes a release. The number of patch authors is thus an indicator of the number of participants in the Linux development process, and the number of committers is an indicator of the amount of manpower that is actively available to begin the integration of patches into a release. Figure 24 shows the number of authors and committers associated with the patches included in each version, both in total and broken down by directory.

For drivers, the numbers of authors and committers are rising at the same rate, roughly at the rate of the increase in the code size. For arch and fs, however, where we have previously noted a higher fault rate (Figures 14 and 15), the number of authors is rising significantly more slowly than the number of committers. The lower number of authors may suggest that potential authors are not able to develop adequate expertise to keep up with the number of new architectures and file systems (Figure 3). The fewer authors engage, the less review there is on code. Finally, the small number of sound authors may explain the previously observed long life of sound faults (Figure 19(a)).

Developer commitment. The more a developer works on the Linux project, the more expertise the developer may be assumed to acquire. We thus study developer commitment as an approximation of developer expertise.

To quantify the commitment of authors and committers, we take the product of the number of commits integrated into the Linux kernel and the period of time (in days) over which the commits have occurred. In doing so, a developer with many recent commits is considered to have the same degree of commitment as a developer with fewer commits over a longer period of time. For instance, an author who gets 12 commits integrated during one year, will have the same degree of commitment as some one who gets 24 commits integrated in 6 months. Figure 25 gives the evolution of the average commitment for each release and for authors and commiters, showing both the value obtained by considering all the commits of that release, and for all the faulty ones, i.e., the commits that introduce one of the faults we found.

Not surprisingly committer commitment is higher than author commitment. Both forms of commitment progress almost constantly over time. This indicates that there is little turnover among the committers and authors. However, for faulty commits, the 


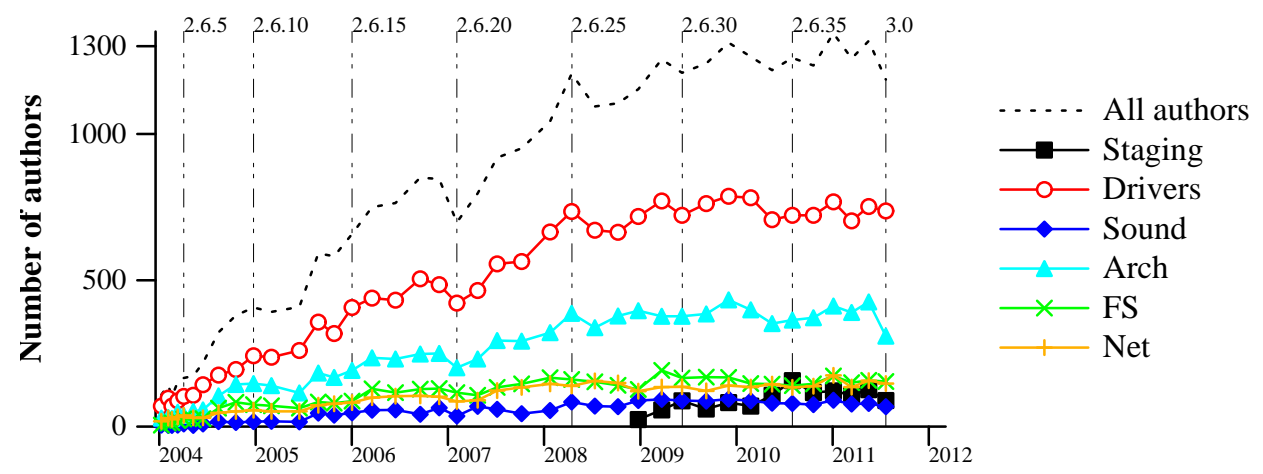

(a) Authors per directory

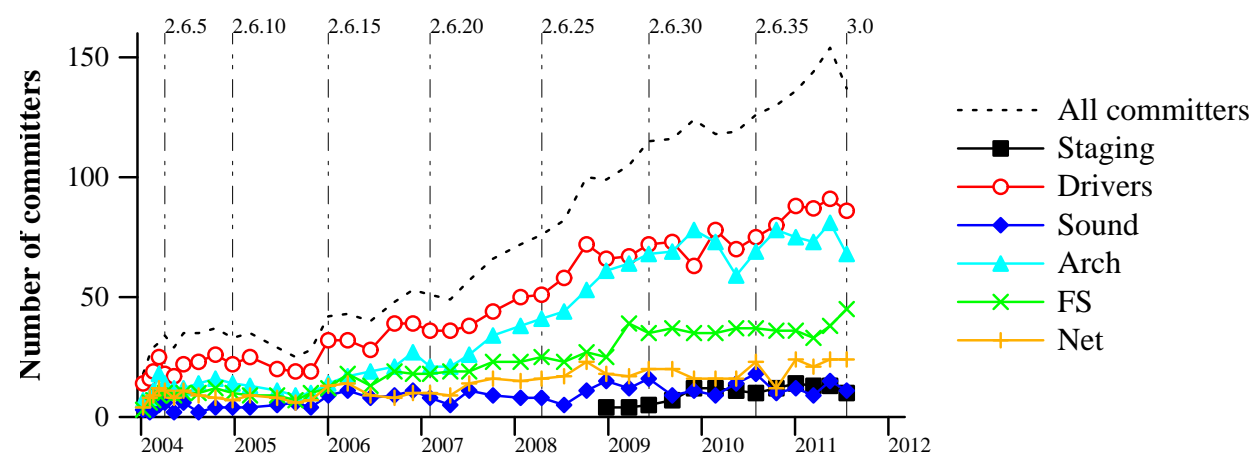

(b) Committers per directory

Fig. 24. Authors and committers per version

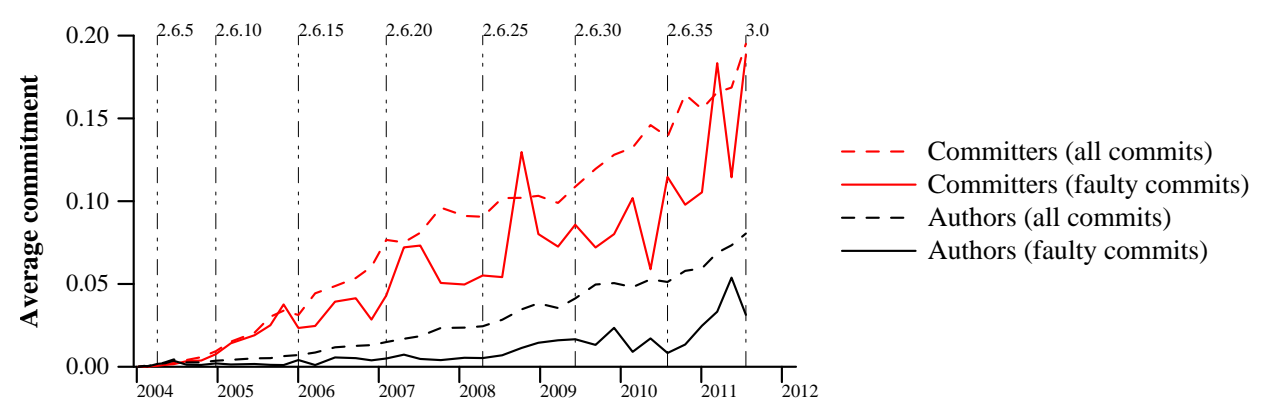

Fig. 25. Developer commitment

average commitment of committers varies greatly even if it is constantly greater than the average commitment of authors. Finally, the average author commitment in the case of faulty commits is significantly lower that the average author commitment for all commits. This indicates that new authors with few commits are more likely to propose faulty patches. As the average committer commitment is also generally lower for faulty commits than the average committer commitment for all commits, these faulty patches are applied.

Figure 26 shows the distribution of the commits according to the commitment of the authors and the committers. Two commitment values are defined for each commit: one for its author, the other for its committer. These commitments are normalized 


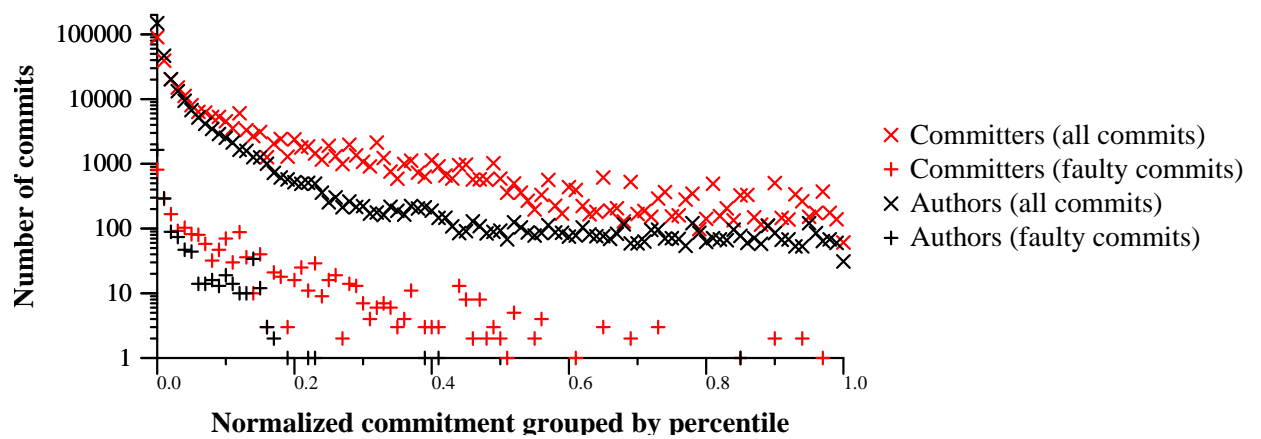

Fig. 26. Developer and maintainer distribution

with respect to the maximal value across the entire period studied, i.e., the computed commitment of Linus Torvalds for the latest commit of Linux 3.0. Finally, each commitment value is rounded and assigned to a percentile of the normalized scale. Commits belonging to the same percentile are grouped and counted. So, each point represents a set of commits with similar author commitment, respectively committer commitment. The size of the set is given on the y-axis.

Once again, for commits in general, the commitment of committers is higher than that of authors, which gives us some confidence in this metric. Concerning faulty commits, more than $99.5 \%$ of them are proposed by authors who rank below $15 \%$. However, the commitment of the committers is more spread across the entire scale.

\subsection{Code structure and evolution}

We consider three metrics: code churn, file age, and function size, and their relation to the number of faults. We also evaluate the quality of Linux code in light of the conjecture that open source code is more reliable because it can be examined by many people.

Churn. Munson and Elbaum [1998] observed that code churn, i.e., the number of times a file is modified, is a good predictor of fault rate. Nagappan and Ball [2005] reached a similar conclusion in a study that used metrics relating to the development of Windows Server 2003 to predict Service Pack 1's fault rate. Figure 27 shows the relationship between the average churn per day preceding the release of each Linux version and the number of the considered faults added per day in that version. The relationship between churn and fault rate is similar. There is an overall tendency of high-churn versions to contain more new faults, even if some high-churn versions have a smaller number of faults than lower churn versions. Recall, however, that in many versions, more faults were eliminated than added; the churn includes the elimination of faults as well.

The solid lines in Figure 27 represent the linear regression of each group of versions and the overall trend. The slope of the trend lines gives the rate of new faults per day as a function of the churn in a day. The legend in the bottom right gives the equations used to produce these lines and the adjusted $R^{2}$ [R Core Team 2014b], as computed by $\mathrm{R}$ [R Core Team 2014a]. Each formula is of the form $y=a x+b$, where $a$ is the slope, and $b$ is 0 , representing the fact that the value at $x=0$, where there is no churn and thus no faults are introduced, is naturally 0 . The adjusted $R^{2}$ represents the goodness of the fit between the model and the actual data. Its value goes up to 1 for a perfect fit, and can be negative, as outliers introduce a penalty. 


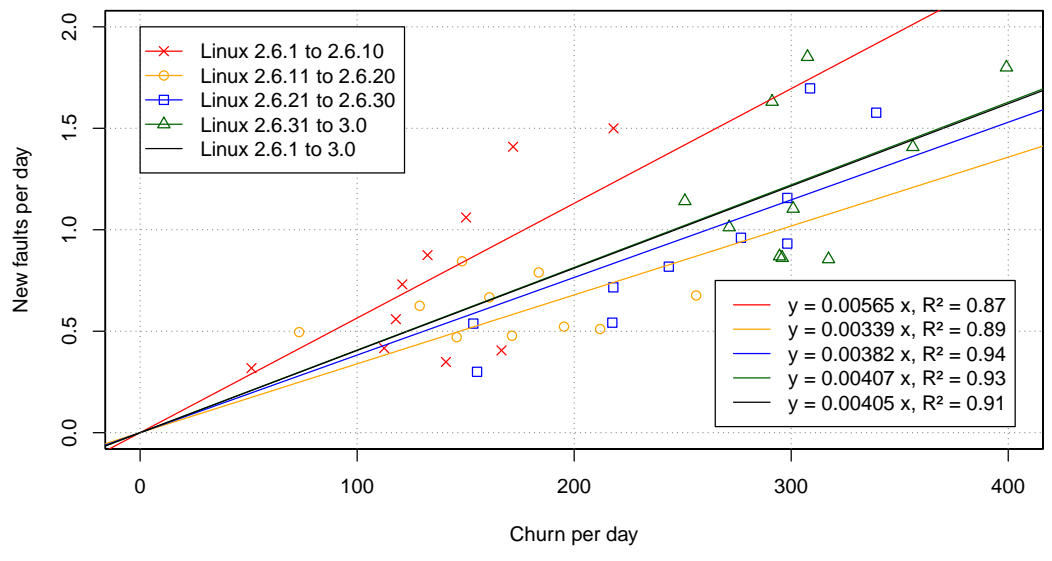

Fig. 27. Churn vs. new faults

The Linux versions are studied in four packets of ten Linux versions grouped by release order. For the first packet, from Linux 2.6.1 to 2.6.10, the rate is the highest with an average churn of 138 modifications per day. In the second packet, the rate has greatly improved and is indeed the lowest of the four ones. At the same time, the churn increased to 168 modifications per day. From Linux 2.6.21 to 2.6.30, the third packet, the rate of new faults per day has increased but the average churn per day in those versions is also higher (251 modifications/day). Finally, in the last packet, the rate of new faults per day has slightly increased, but it is now almost identical to the overall rate between Linux 2.6.1 and 3.0. However, the churn per day has further increased to 309 modifications / day.

File age and function size. One may expect that as a file ages the number of faults would decrease. One may also expect that large functions would be more complicated and thus tend to harbor more faults. Indeed, Chou et al. found these trends in Linux 2.4.1, to a varying degree for the different fault kinds. Figure 28 considers the relationship between file age or function size and fault rate in Linux 3.0. Files and functions are organized by increasing age or size, respectively, then collected into buckets containing an exponentially decreasing number of elements, from the smallest age or size to the largest ( $c f$. Section 4.3). This strategy permits a fine degree of granularity for the files with greater ages or sizes, respectively, without degrading the results for the smaller ages and sizes. Note that we change the bucket strategy with respect to the file age, compared to Figure 8 , page 15 . We thus reflect the changes in the file age distribution, as shown in Table $\bar{V}$, and the observations of Figure 2, page 9, Each graph shows the average age or size of the files or functions in each bucket and their average fault rate.

Table V. File age distribution for Linux 3.0

\begin{tabular}{|l|c|c|c|c|c|c|c|c|c|}
\hline Age (in years) & 0 & 1 & 2 & 3 & 4 & 5 & 6 & 7 & 8 \\
\hline Number of files & 5,934 & 3,826 & 7,989 & 2,220 & 1,943 & 1,522 & 858 & 638 & 1,487 \\
\hline \hline Age (in years) & 9 & 10 & 11 & 12 & 13 & 14 & 15 & 16 & 17 \\
\hline Number of files & 1,276 & 265 & 662 & 301 & 311 & 251 & 208 & 123 & 241 \\
\hline
\end{tabular}

Figure 28(a) shows that in Linux 3.0, the youngest half of the files, represented by the leftmost point, has a fault rate about twice that of the next youngest quarter of the files. For older files, however, the relation between age and fault rate is less clear, 


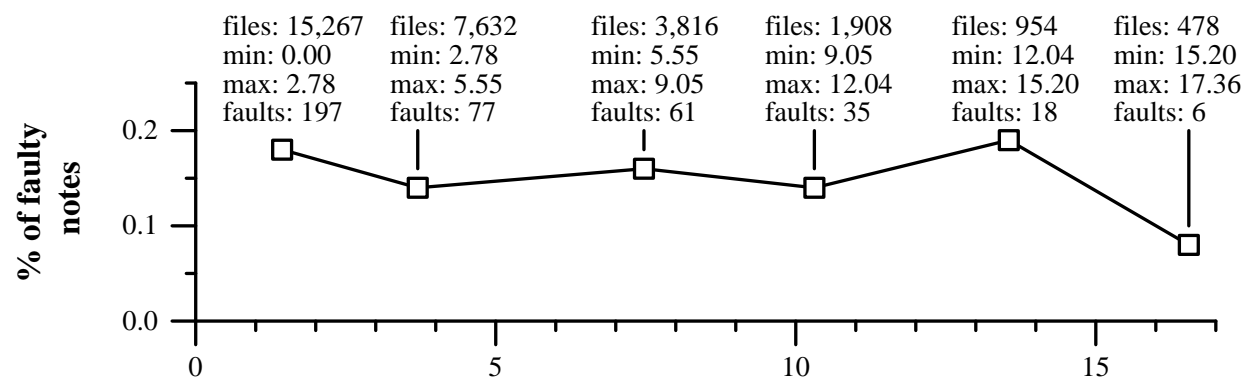

File age in years

(a) Fault rate by file age in Linux 3.0

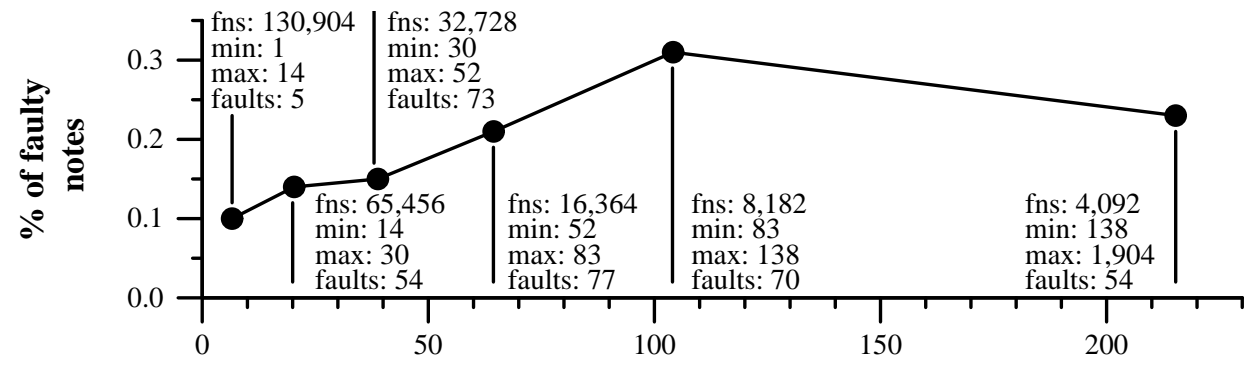

Function size in number of lines

(b) Fault rate by function size in Linux 3.0

Fig. 28. Impact of file age and function size. Each point represents twice as many files or functions as the next point. At each point, "files" and "fns" refer respectively to the number of files and functions considered to compute the point, "min" and "max" refer to the minimal or maximal age or size represented by the point, and faults indicates the number of faults that occur in the code in that range.

as the rate first increases and then decreases as the file age increases. On the other hand, the average fault rate clearly increases as the function size increases (Figure 28(b)). Indeed, the bucket with the smallest functions (up to 14 lines) has a significantly lower fault rate than the next buckets. There is also an increase up to 138 lines. This suggests that large functions, with about a hundred lines of code, need more attention than others, even if they represent only $3 \%$ of the functions. $3 \%$ of the functions in Linux 3.0 , however, amounts to about 8,000 functions, making them difficult to check exhaustively. Tool support is thus crucial.

For Linux 2.4.1, we observed in Figure 6(c) (page 14) that the functions found in the fourth bucket had the highest fault rate at $1.1 \%$ of the faulty notes. Compared to Linux 2.4.1, the maximum is for larger functions in Linux 3.0, and in the fifth bucket. This maximum has dropped to $0.3 \%$ of the faulty notes. Finally, as observed for Linux 2.4.1, the largest functions, represented by the rightmost bucket, have a slightly smaller fault rate than those of around 100 lines.

Kernel configuration. In "The Cathedral and the Bazaar" [Raymond 2001], Eric S. Raymond formalized Linus' law: "given enough eyeballs, all bugs are shallow." But, in practice, code that is frequently executed, or at least frequently compiled, is more likely to be reviewed than the rest. When code is frequently executed, many users are likely to encounter any faults and some may fix the faults themselves or submit a request that the faults be fixed by a kernel maintainer. When code is frequently compiled, 


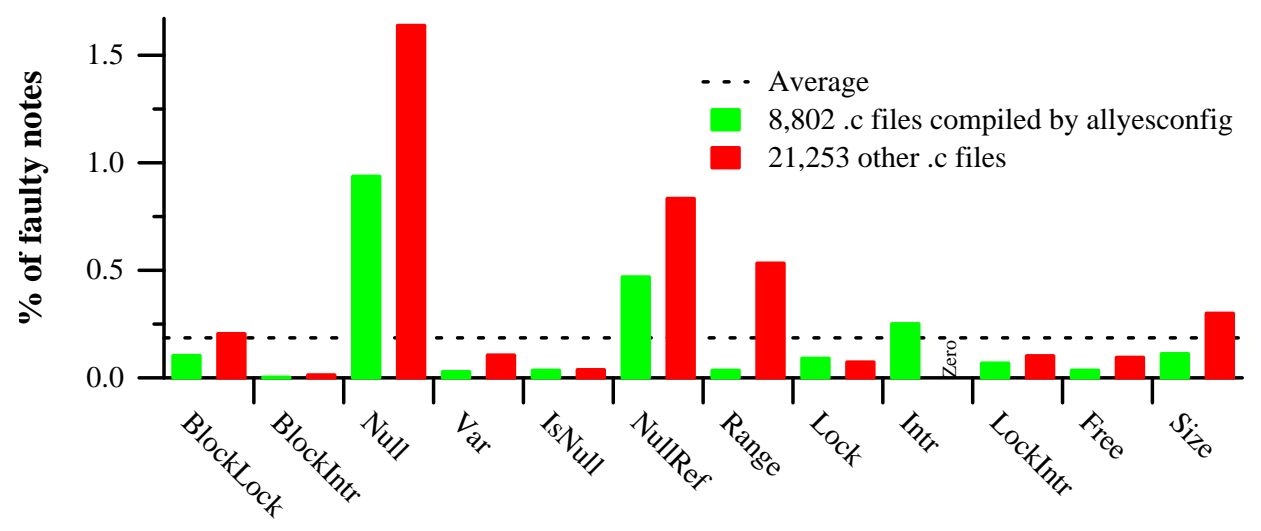

Fig. 29. Fault rate compared between configurations (Linux 3.0)

even if it is not frequently executed, it can easily be submitted to fault-finding tools that are integrated with the kernel compilation process. "Eyeballs" may also focus on fixing faults in code that they are able to compile, as even standard compilers such as gcc perform some sanity checks that provide some confidence that a fix has not e.g., introduced a typographical error, even if the code cannot be tested.

Figure 29 compares the number of faults found in the .c files that are compiled using the configuration generated on an x86 architecture by the Linux Makefile argument allyesconfig to the number of faults found in the .c files found in the rest of the Linux kernel 111 The Makefile argument allyesconfig creates a configuration file for the given architecture that includes as many options as possible without causing a conflict and without including drivers/staging. Thus, it can be assumed to trigger the compilation of a set of well-tested files and a superset of what is normally included with a Linux distribution, and thus what is executed by ordinary users. In most cases, we do find that the allyesconfig files have a lower fault rate than the other files. The only exceptions are for Lock and Intr faults, but there is only a single fault in the case of Intr. For Lock, 13 out of 15 Lock faults are in files included in the allyesconfig configuration, and this configuration contains only 5 times more Lock notes than the other files. The fault rate of Lock notes in allyesconfig files is thus higher than that in other files.

\section{TOWARDS NEW CODE VERSIONS}

In this section, we first consider some new checkers related to the use of the RCU locking API, which was introduced in 2.5.43, about one year before the release of 2.6.0. Then, we consider how our experimental protocol eases the extension of the results to new Linux versions.

\subsection{How does the fault rate in new APIs compare}

The fault kinds that we have considered until now involve code structures that are either common to all $\mathrm{C}$ code or that have been present in Linux for a long time. To give an alternate perspective on the robustness of Linux 2.6 code, we consider the use of a more recent and specialized locking API: the one that implements the ReadCopy-Update (RCU) mechanism [McKenney and Walpole 2008]. RCU is a lightweight synchronization mechanism that protects readers against writers. Reads are wait-free

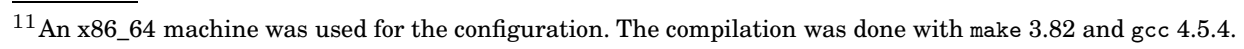




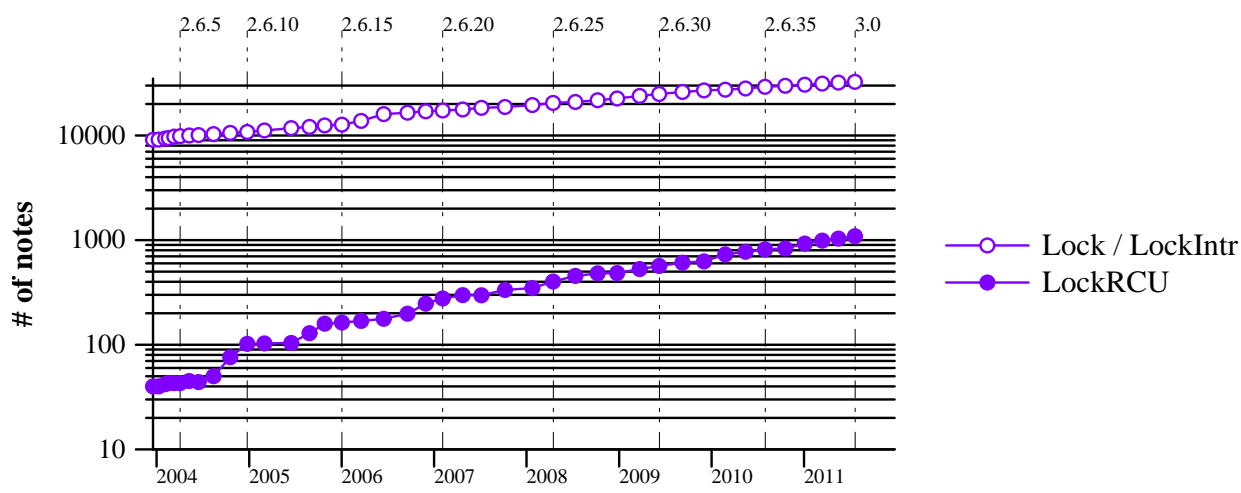

Fig. 30. The use of spinlock and mutex locking functions and the use of RCU locking functions

with very low overhead while writes are more expensive. Therefore, RCU particularly favors workloads that mostly read shared data rather than updating it. RCU has been increasingly used in Linux 2.6, but is still used less often than spinlocks and mutexes, as shown in Figure 30.

The main functions that we consider in the RCU API are the locking functions: rcu_read_lock, srcu_read_lock, rcu_read_lock_bh, rcu_read_lock_sched, and rcu_read_lock_sched_notrace. We identify as faults any cases where a blocking function is called while a RCU lock is held (BlockRCU) and where a RCU lock is taken but not released (LockRCU). These are analogous to the previously considered BlockLock and Lock fault kinds, respectively. Double-acquiring an RCU lock is allowed, and thus this is not considered to be a fault. Finally, we identify as a fault any case where shared data is accessed using $\mathrm{rcu}_{-}$dereference when an RCU lock is not held (DerefRCU).

The largest number of uses of the RCU lock functions is in net, followed by drivers in versions earlier than 2007, and other in later versions, with kernel, security and $\mathrm{mm}$ being the main directories in the other category where it is used. Correspondingly, as shown in Figure 31, most faults are found in net, and the few remaining faults are found in other. The average lifespan of the net faults is around one year, while that of the other faults is a few months longer. As shown in Figure 32, most of the faults are BlockRCU faults, and the largest number of faults per version is only 13, in Linux 2.6.37. Thus, overall, the fault rates are substantially below the fault rates observed for any of the previously considered fault kinds, and in particular far below the rates observed for the various locking faults (Lock, Intr, and LockIntr). This may be explained by the fact that the developers who have added calls to the various RCU locking functions are relatively experienced, having at the median 123 patches accepted between Linux 2.6.12 and Linux 2.6.33. Still, these results suggest that Linux developers can be successful at adopting a new API and using it correctly.

\subsection{Processing New Versions}

As presented in Section 2, the automatic report correlations provided by Herodotos make it easy to extend an existing set of results to the next version of Linux. As an experiment, we have studied the extent to which the annotated reports for Linux 2.6.39 can help evaluate the reports for Linux 3.0. For Linux 3.0, as shown in Figure 33, the BlockLock checker produces 161 reports of potential faults of which 121 are faults inherited from Linux 2.6.39, and 37 are false positives also present in that version. Herodotos would annotate these reports automatically, leaving only three reports to be annotated by the user. In this case, the three reports are faults. Overall, for Linux 


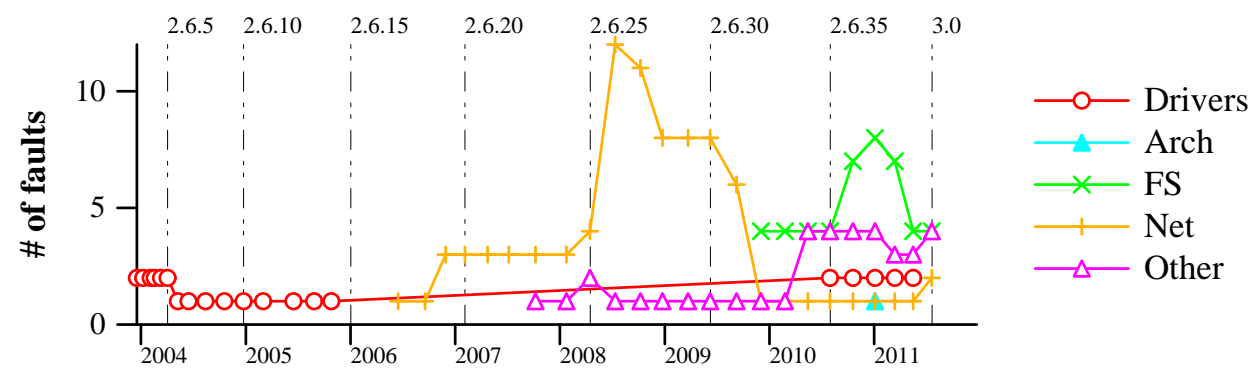

Fig. 31. RCU faults per directory

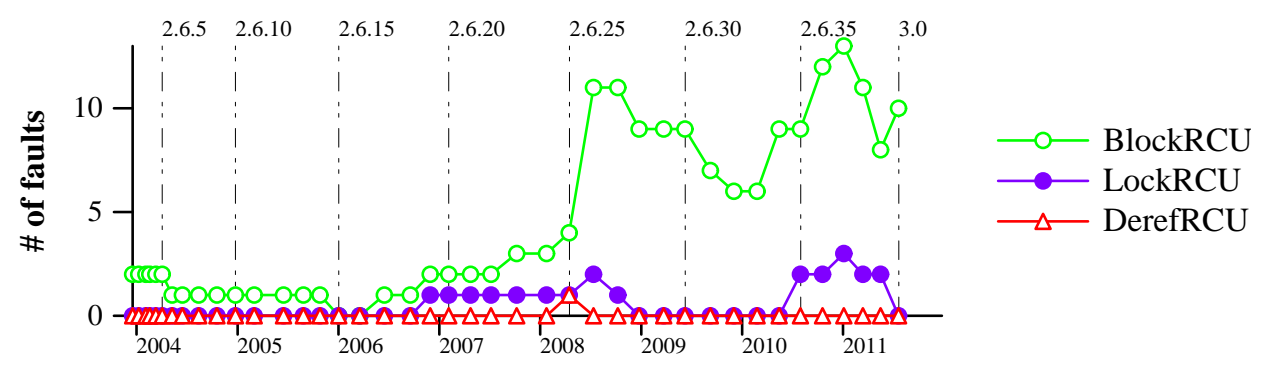

Fig. 32. RCU faults through time

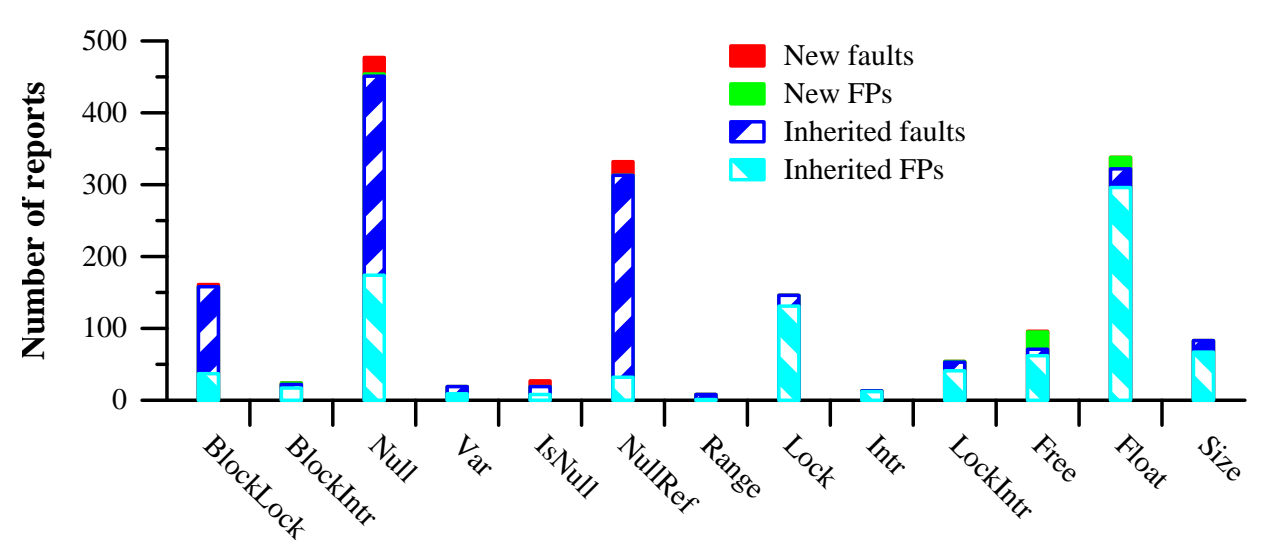

Fig. 33. Reports generated for Linux 3.0

3.0 there are 100 new reports to consider. Most of these can be dealt with in a few minutes each.

\section{LIMITATIONS}

The main limitations of our work are in the choice of faults considered and the definition of the checkers. We have focused on the same kinds of faults as Chou et al., to be able to assess the changes in Linux since their work. Current fault finding tools, including Coccinelle, are able to find other kinds of faults, such as memory leaks. The considered set of faults also does not include concurrency faults, which are becoming increasingly important with the increasing prevalence of multicore architectures. A recent study of concurrency faults in infrastructure software, however, has shown 
that over $20 \%$ of deadlocks are caused by a thread reacquiring a resource it already holds [Lu et al. 2008], amounting to a double lock, as detected by our Lock checker.

Our checkers could also be improved to reduce the number of false positives. For instance, the Float checker generates a large number of false positives, as shown for Linux 3.0 in Figure 33 in the previous section. Some kinds of false positives are due to recurring patterns specific to certain Linux files. Taking these patterns into account in the checkers would avoid generating large numbers of trivial false positive reports. Finally, we have tried to be conservative in our identification of real faults, and this may have lead to an underestimation of their number. By making our results available in a public archive, we hope to benefit from feedback from the Linux community to improve our classification strategies.

\section{RELATED WORK}

Chou et al. [2001] briefly considered OpenBSD, as well as Linux. Because of its wider use and more active development, we have focused only on Linux instead, comparing the properties of old and new versions.

In previous work, Palix et al. have used Coccinelle to conduct a study of faults in versions of Linux and several other open-source projects released between 2005 and 2009 [Palix et al. 2010a]. They proposed Herodotos, in order to correlate the faults between releases. They did not consider fault kinds requiring an interprocedural analysis, nor did they consider lock-related faults. As shown in Figure 11, these are among the most prevalent and also have high impact. Lawall et al. [2013] proposed a methodology for finding interprocedural faults in Linux code, but consider only a single Linux version.

Israeli and Feitelson [2010] have studied 810 versions of the Linux kernel, from Linux 1.0 to Linux 2.6.25. They considered traditional source-code metrics to measure complexity [McCabe 1976] and maintainability [Oman and Hagemeister 1994], rather than actual numbers of faults. They found that the complexity per Linux function is decreasing, and the maintainability is increasing. Nevertheless, they did find that arch and drivers contain some very high complexity functions, typically interrupt handlers or ioctl functions, and that arch and drivers code is somewhat less maintainable than the code in other parts of the kernel. Their work is complementary to ours, and reaches some of the same conclusions.

Song et al. [2010] have studied the reasons for software hangs in open-source infrastructure software, such as MySQL and Apache. They focused on bug reports rather than analysis of the source code. They found that most types of concurrency faults are fixed on average within 100 days. Nevertheless, they did not take into account the time elapsed between the introduction of the fault and the time when it was first detected, and so the actual fault lifetime may be more in line with what we have observed. Lu et al. [2008] also considered concurrency faults in infrastructure software, primarily focusing on the kinds of tools that would be helpful to address them.

UNDERTAKER [Tartler et al. 2012; Nadi et al. 2013] finds inconsistencies between the Linux configuration files and the associated conditional compilation directives (e.g., \#ifdef) present in the code. The faults that they consider are thus orthogonal to the ones considered here. Using Herodotos, they found an average fault lifespan of 6 Linux releases [Nadi et al. 2013]. However, they do not seem to have used the Kaplan-Meier estimator, and thus the censoring of the last version studied does not seem to be correctly handled. As UNDERTAKER focuses on configuration faults and Coccinelle focuses on code faults, it would be interesting to integrate the results obtained by the tool in the future, to obtain a more complete view of the quality of Linux code.

Hector [Saha et al. 2013] is a tool that checks protocol usage and reports missing function calls in error-handling code. It thus finds a narrower range of faults than 
those considered here, but it includes a dataflow analysis that allows it to collect more precise information than Coccinelle. It could be interesting to conduct a similar study of the history of the kinds of faults found using Hector.

We have noted that file systems have a higher fault rate than drivers in some recent Linux versions (Figure 14, in Section 5.2). Some recent work has focused on issues in file systems. Rubio-González et al. [2009] study the use of error codes in file systems. Lu et al. [2013] study the patches applied to six major file systems, to understand the file systems' evolution. Part of this study includes categorization of the kinds of faults that are fixed by these patches; some faults correspond to the kinds that we have considered, however they consider only the faults that have been fixed, while our focus is on those that remain in the code. Yang et al. [2006] applied model checking to find a small number of faults in file system code, including some severe ones. More recently, Keller et al. [2013] have proposed a methodology for designing a file system that is formally proved correct. Our work is largely complementary to these studies.

\section{CONCLUSION}

During the last 10 years, much of the research in operating system reliability has been predicated on the assumption that drivers are the main problem. The first major result of our study is that while drivers still has the largest number of faults in absolute terms, it no longer has the highest fault rate in Linux kernel code, having been supplanted by the HAL. The second major result of our study is that even though faults are continually being introduced, the overall code quality is improving. Our work thus shows the importance of being able to periodically repeat the study of faults in source code in order to revise research priorities as the fault patterns change in response to research and development efforts for improving the quality of Linux code. Because the priorities of individuals and institutions change over time, the need to repeat such studies implies that the tools and other data required must be available in an open archival repository.

Our study also shows that tools, while used, are under-exploited. Tools are indeed available to find all of the fault kinds considered in this paper. The fact that these kinds of faults remain and have a relatively long lifespan suggests that research is needed on how to design tools that are better integrated into the Linux development process. Another potential problem is the reactiveness of maintainers. Indeed, some services have no maintainer, but remain in the kernel source tree. This may somewhat artificially increase the number of faults. Still, any such faults can impact anyone who uses the affected code.

Our study has identified 855 faults in Linux 3.0, including RCU faults, some of which have not yet been corrected in the current developer snapshot, linux-next, as of November 5, 2013. We have submitted a number of patches based on our results.

\section{ACKNOWLEDGMENTS}

We thank the anonymous reviewers for their feedback. We also thank Emmanuel Cecchet, Willy Zwaenepoel and Frans Kaashoek for comments on an earlier version of this paper. Finally, we thank Nicolas Glade, Adeline Leclercq Samson and Frédérique Letué for their feedback on the Kaplan-Meier estimator.

\section{REFERENCES}

Alex Aiken, Suhabe Bugrara, Isil Dillig, Thomas Dillig, Brian Hackett, and Peter Hawkins. 2007. An overview of the Saturn project. In Proceedings of the 7th ACM SIGPLAN-SIGSOFT Workshop on Program Analysis for Software Tools and Engineering, PASTE'07. ACM, San Diego, CA, 43-48.

Christian Bird, Nachiappan Nagappan, Premkumar Devanbu, Harald Gall, and Brendan Murphy. 2009. Does distributed development affect software quality? an empirical case study of windows vista. In 
31st International Conference on Software Engineering. IEEE, Vancouver, British Columbia, Canada, $518-528$.

Julien Brunel, Damien Doligez, René Rydhof Hansen, Julia Lawall, and Gilles Muller. 2009. A foundation for flow-based program matching using temporal logic and model checking. In The 36th annual ACM SIGPLAN-SIGACT Symposium on Principles of Programming Languages. ACM, Savannah, GA, USA, $114-126$.

CDHSE 2009. How is the department of homeland security involved? (2009). http://scan.coverity.com/faq.html\#how-department-homeland-security-involved

Checkpatch 2006. Checkpatch. (2006). http://www.codemonkey.org.uk/projects/checkpatch/.

Andy Chou, Junfeng Yang, Benjamin Chelf, Seth Hallem, and Dawson Engler. 2001. An empirical study of operating systems errors. In Proceedings of the 18th ACM Symposium on Operating System Principles. ACM, Banff, Canada, 73-88. http://www.stanford.edu/ engler/

Comedi 1999. Comedi: Linux control and measurement device interface. (1999). http://www.comedi.org/

Jonathan Corbet. 2010a. The age of kernel code in various subsystems. (Feb. 2010). http://lwn.net/Articles/374622/

Jonathan Corbet. 2010b. How old is our kernel? (Feb. 2010). http://wn.net/Articles/374574/

Coverity 2008. Static source code analysis, static analysis, software quality tools by Coverity Inc. http://www.coverity.com// (2008).

Alex Depoutovitch and Michael Stumm. 2010. Otherworld - giving applications a chance to survive OS kernel crashes. In EuroSys. ACM, Paris, France, 181-194.

Fedora 2013. Fedora project. (2013). http://fedoraproject.org/

Jorrit N. Herder, Herbert Bos, Ben Gras, Philip Homburg, and Andrew S. Tanenbaum. 2009. Fault isolation for device drivers. In The 39th Annual International Conference on Dependable Systems and Networks (DSN). IEEE/IFIP, Estoril, Portugal, 33-42.

IEEE Std 982.2 1988. IEEE guide for the use of IEEE standard dictionary of measures to produce reliable software. (1988).

Ayelet Israeli and Dror G. Feitelson. 2010. The Linux kernel as a case study in software evolution. Journal of Systems and Software 83, 3 (2010), 485-501.

Yujuan Jiang, Bram Adams, and Daniel M. Germán. 2013. Will my patch make it? and how fast?: case study on the Linux kernel. In Proceedings of the 10th Working Conference on Mining Software Repositories, MSR '13. IEEE/ACM, San Francisco, CA, USA, 101-110.

Edward L Kaplan and Paul Meier. 1958. Nonparametric estimation from incomplete observations. Journal of the American statistical association 53, 282 (1958), 457-481.

Gabriele Keller, Toby Murray, Sidney Amani, Liam O'Connor, Zilin Chen, Leonid Ryzhyk, Gerwin Klein, and Gernot Heiser. 2013. File systems deserve verification too!. In 7th Workshop on Programming Languages and Operating Systems (PLOS 2013). Nemacolin Woodlands Resort, PA, USA, 7.

Julia L. Lawall, Julien Brunel, Nicolas Palix, René Rydhof Hansen, Henrik Stuart, and Gilles Muller. 2013. WYSIWIB: exploiting fine-grained program structure in a scriptable API-usage protocol-finding process. Software: Practice and Experience 43, 1 (Jan. 2013), 67-92.

Zhenmin Li and Yuanyuan Zhou. 2005. PR-Miner: automatically extracting implicit programming rules and detecting violations in large software code. In Proceedings of the 10th European Software Engineering Conference held jointly with 13th ACM SIGSOFT International Symposium on Foundations of Software Engineering. ACM, Lisbon, Portugal, 306-315.

LKML 2013. Lkml: The Linux kernel mailing list. http://kml.org/ (2013).

Lanyue Lu, Andrea C. Arpaci-Dusseau, Remzi H. Arpaci-Dusseau, and Shan Lu. 2013. A study of Linux file system evolution. ;login: The USENIX Magazine 38, 3 (June 2013), 10-17.

Shan Lu, Soyeon Park, Eunsoo Seo, and Yuanyuan Zhou. 2008. Learning from mistakes: a comprehensive study on real world concurrency bug characteristics. In Architectural Support for Programming Languages and Operating Systems (ASPLOS). ACM, Seattle, WA, USA, 329-339.

T. J. McCabe. 1976. A complexity measure. IEEE Transactions on Software Engineering 2, 4 (July 1976), $308-320$.

Paul E. McKenney and Jonathan Walpole. 2008. Introducing technology into the Linux kernel: a case study. ACM SIGOPS Operating Systems Review 42, 5 (2008), 4-17.

John C. Munson and Sebastian G. Elbaum. 1998. Code churn: A measure for estimating the impact of code change. In International Conference Software Maintenance (ICSM). IEEE, Bethesda, Maryland, USA, $24-31$. 
Sarah Nadi, Christian Dietrich, Reinhard Tartler, Richard C. Holt, and Daniel Lohmann. 2013. Linux variability anomalies: what causes them and how do they get fixed?. In Proceedings of the 10th Working Conference on Mining Software Repositories, MSR '13. IEEE/ACM, San Francisco, CA, USA, 111-120.

Nachiappan Nagappan and Thomas Ball. 2005. Use of relative code churn measures to predict system defect density. In 27th International Conference on Software Engineering (ICSE). ACM, St. Louis, Missouri, USA, 284-292.

Paul Oman and Jack Hagemeister. 1994. Construction and testing of polynomials predicting software maintainability. Journal of Systems and Software 24, 3 (1994), 251-266.

Org 2013. Org-mode homepage. http://orgmode.org/. (2013).

Yoann Padioleau, Julia Lawall, René Rydhof Hansen, and Gilles Muller. 2008. Documenting and automating collateral evolutions in Linux device drivers. In EuroSys 2008. ACM, Glasgow, Scotland, 247-260.

Nicolas Palix, Julia Lawall, and Gilles Muller. 2010a. Tracking code patterns over multiple software versions with Herodotos. In Proc. of the ACM International Conference on AspectOriented Software Development, AOSD'10. ACM, Rennes and Saint Malo, France, 169-180. DOI : http://dx.doi.org/10.1145/1739230.1739250

Nicolas Palix, Suman Saha, Gaël Thomas, Christophe Calvès, Julia Lawall, and Gilles Muller. 2010b. Website of Faults in Linux: Ten years later. (Dec. 2010). http://faultlinux.lip6.fr/

Nicolas Palix, Suman Saha, Gaël Thomas, Christophe Calvès, L. Lawall, Julia, and Gilles Muller. 2010c. Faults in Linux: Ten Years Later. Research Report RR-7357. INRIA. 21 pages. http://hal.inria.fr/inria-00509256

Nicolas Palix, Gaël Thomas, Suman Saha, Christophe Calvès, Julia Lawall, and Gilles Muller. 2011. Faults in Linux: Ten years later. In Sixteenth International Conference on Architectural Support for Programming Languages and Operating Systems (ASPLOS 2011). ACM, Newport Beach, CA, USA, 305-318.

R Core Team. 2014a. The R project for statistical computing. (April 2014). http://www.r-project.org/

R Core Team. 2014b. Summarizing linear model fits. (April 2014). http://stat.ethz.ch/R-manual/R-patched/library/stats/html/summary.lm.html

Eric S. Raymond. 2001. The Cathedral and the Bazaar: Musings on Linux and Open Source by an Accidental Revolutionary. O’Reilly \& Associates, Inc.

Cindy Rubio-González, Haryadi S. Gunawi, Ben Liblit, Remzi H. Arpaci-Dusseau, and Andrea C. ArpaciDusseau. 2009. Error propagation analysis for file systems. In PLDI. ACM, Dublin, Ireland, 270-280.

Suman Saha, Jean-Pierre Lozi, Gaël Thomas, Julia L Lawall, and Gilles Muller. 2013. Hector: Detecting resource-release omission faults in error-handling code for systems software. In Dependable Systems and Networks (DSN), 2013 43rd Annual International Conference on. IEEE/IFIP, Budapest, Hungary, $1-12$.

D. Searls. 2004. Sparse, Linus \& the Lunatics. (Nov. 2004). Available at http://www.linuxjournal.com/article/7272.

Xiang Song, Haibo Chen, and Binyu Zang. 2010. Why software hangs and what can be done with it. In International Conference on Dependable Systems and Networks (DSN 2010). IEEE/IFIP, Chicago, IL, USA, 311-316.

Sparse 2003. Sparse. (2003). https://sparse.wiki.kernel.org/.

Brad Spencer. 2009. Local kernel exploit in /dev/net/tun. (July 2009). http://grsecurity.net/ spender/exploits/cheddar_bay.tgz.

Michael M. Swift, Muthukaruppan Annamalai, Brian N. Bershad, and Henry M. Levy. 2006. Recovering device drivers. ACM Transactions on Computer Systems 24, 4 (2006), 333-360.

Reinhard Tartler, Julio Sincero, Christian Dietrich, Wolfgang Schröder-Preikschat, and Daniel Lohmann. 2012. Revealing and repairing configuration inconsistencies in large-scale system software. International Journal on Software Tools for Technology Transfer 14, 5 (2012), 531-551.

The Kernel Janitors. 2010. Smatch, the source matcher. (2010). Available at http://smatch.sourceforge.net

Ubuntu 2013. Ubuntu. (2013). http://www.ubuntu.com/

Xi Wang, Nickolai Zeldovich, M. Frans Kaashoek, and Armando Solar-Lezama. 2013. Towards optimizationsafe systems: analyzing the impact of undefined behavior. In Proceedings of the Twenty-Fourth ACM Symposium on Operating Systems Principles (SOSP '13). ACM, Farminton, Pennsylvania, 260-275.

David Wheeler. 2006. Flawfinder home page. Web page: http://www.dwheeler.com/flawfinder/. (Oct. 2006). http://www.dwheeler.com/flawfinder/

David A. Wheeler. 2013. SLOCCount. (2013). http://www.dwheeler.com/sloccount/. 
Junfeng Yang, Paul Twohey, Dawson Engler, and Madanlal Musuvathi. 2006. Using model checking to find serious file system errors. ACM Trans. Comput. Syst. 24, 4 (Nov. 2006), 393-423. DOI :http://dx.doi.org/10.1145/1189256.1189259

\section{A. APPENDIX}

\section{A.1. Lock and interrupt functions}

Locking functions: \{mutex,spin,read,write\}_lock, \{mutex,spin,read,write\}_trylock

Interrupt disabling functions:. cli, local_irq_disable

Functions combining

both::\{read,write,spin\}_lock_irq, $\{$ read,write,spin\}_lock_irqsave, local_irq_save, save_and_cli 